\title{
Growth of non-linear structures and spherical collapse in the Galileon Ghost Condensate model
}

\author{
Noemi Frusciante ${ }^{\mathrm{a}}$, Francesco Pace ${ }^{\mathrm{b}}$ \\ ${ }^{a}$ Instituto de Astrofísica e Ciências do Espaço, Faculdade de Ciências da Universidade de Lisboa, \\ Edificio C8, Campo Grande, P-1749016, Lisboa, Portugal \\ ${ }^{b}$ Jodrell Bank Centre for Astrophysics, School of Natural Sciences, Department of Physics and Astronomy, \\ The University of Manchester, Manchester, M13 9PL, U.K.
}

\begin{abstract}
We present a detailed study of the collapse of a spherical matter overdensity and the non-linear growth of large scale structures in the Galileon ghost condensate (GGC) model. This model is an extension of the cubic covariant Galileon (G3) which includes a field derivative of type $\left(\nabla_{\mu} \phi \nabla^{\mu} \phi\right)^{2}$ in the Lagrangian. We find that the cubic term activates the modifications in the main physical quantities whose time evolution is then strongly affected by the additional term. Indeed, the GGC model shows largely mitigated effects in the linearised critical density contrast, non-linear effective gravitational coupling and the virial overdensity with respect to G3 but still preserves peculiar features with respect to the standard $\Lambda$ CDM cosmological model, e.g., both the linear critical density contrast and the virial overdensity are larger than those in $\Lambda$ CDM. The results of the spherical collapse model are then used to predict the evolution of the halo mass function, non-linear matter and lensing power spectra. While at low masses the GGC model presents about $10 \%$ fewer objects with respect to $\Lambda \mathrm{CDM}$, at higher masses for $z>0$ it predicts $10 \%(z=0.5)-20 \%(z=1)$ more objects per comoving volume. Using a phenomenological approach to include the screening effect in the matter power spectrum, we show that the difference induced by the modifications of gravity are strongly dependent on the screening scale and that differences can be up to $20 \%$ with respect to $\Lambda \mathrm{CDM}$. These differences translate to the lensing power spectrum where qualitatively the largest differences with respect to the standard cosmological model are for $\ell<10^{3}$. Depending on the screening scale, they can be up to $25 \%$ on larger angular scales and then decrease for growing $\ell$. These results are obtained for the best fit parameters from linear cosmological data for each model.
\end{abstract}

Keywords: modified gravity, Vainshtein mechanism, spherical collapse, mass function, matter \& lensing power spectra

\section{Introduction}

The late-time acceleration of the Universe has been confirmed by several cosmological observations [1-8]. Its modelling within General Relativity (GR) is done through the cosmological constant $\Lambda$ which counteracts the attractive force of gravity realising the desired acceleration. The resulting model is the $\Lambda$-cold-dark-matter $(\Lambda \mathrm{CDM})$ which provides an accurate picture of the Universe. However, it still contains a number of open theoretical issues [9] which might signal the breakdown of GR. Alternative proposals, known as modified gravity theories (MG), suggest to modify the gravitational interaction on cosmological scales. The latter usually foresee the inclusion of additional degrees of freedom (dofs) [9-20]. Among these proposals, scalar-tensor theories of gravity have played a prominent role as they simply add a scalar dof to the usual tensor modes of GR [9, 14,-16, 18, 20,-25]. For example, Horndeski theory (or Galileon theory) [21, 23, 26], is described by an action characterized by four free functions of the scalar field $\phi$ and its kinetic energy $X=\nabla_{\mu} \phi \nabla^{\mu} \phi$. In this theory, the scalar field

Email addresses: nfrusciante@fc.ul.pt (Noemi Frusciante), francesco.pace@manchester.ac.uk (Francesco Pace) obeys a second order Euler-Lagrange equation and a fixed form for these functions defines a model. In the last decade several Galileon models have been proposed [27,-34] and some of them have been tested against cosmological data at linear level [35[42]. The so-called Galileon ghost condensate model (GGC) $[28,31]$ is of particular interest as it is the first Galileon model to be statistically preferred by data over $\Lambda$ CDM [40]. This is due to a suppression in the low- $\ell$ tail of the Cosmic Microwave Background (CMB) temperature-temperature power spectrum with respect to $\triangle \mathrm{CDM}$ and a peculiar evolution of the expansion history, characterised by a dark energy (DE) equation of state $w_{\mathrm{DE}}$ entering the region $(-2,-1)$ during the matter era without ghosts.

The GGC model possesses a screening mechanism, dubbed Vainshtein mechanism [9, 43, 46], which suppresses the modifications to gravity on Solar-System scales where GR is tested with exquisite precision [47, 48]. The Vainshtein mechanism operates through the second derivative of the scalar field $\partial^{2} \phi$, dropping the modification to the gravity force in high-density environment. Screening mechanisms play a very important role when considering the formation of gravitationally bound structures: indeed, during the collapse phase the density of the region can be sufficiently high to significantly modify the dynam- 
ics of the scalar field. Analysis in this direction have been performed using the spherical collapse model for Galileon models [49-52]. For example, in DGP braneworld gravity, the Vainshtein mechanism affects both force and energy conditions during collapse, in particular the conservation of the Newtonian total energy is violated [49] and in both DGP and cubic Galileon models an enhancement of structure formation is found due to a screening mechanism which is not effective until late time in the collapse [49, 52]. Hence, in order to properly constrain any MG model, it is important to fully understand the impact the screening mechanism has on the dynamics of the scalar field at non-linear scales. This investigation is timely and relevant in light of new and high quality data in the non-linear regime of weak lensing and galaxy clustering from upcoming surveys,

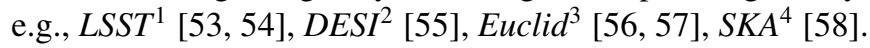

In this paper we aim at investigating how the effects of nonlinearities in the GGC scenario change the collapse process of a spherical overdensity and what the role of the Vainshtein mechanism is. We will then use the results of the analysis of the spherical collapse model to make theoretical predictions on the abundances of halos and discuss the non-linear matter and lensing power spectra.

The work is organised as follows. In Section 2 we introduce the GGC model and give an overview of the background equations. In Section 3 we present the linearly perturbed equations and the evolution of the linear matter density perturbation and its growth rate. Then, in Section 4 we derive the non-linear corrections to the equations for both the scalar field and matter perturbations. The spherical collapse is then studied in Section 5 In Section 6 we present the theoretical predictions for the non-linear matter and lensing power spectra, and in Section 7 the effects of the GGC model on the abundances of halos. We finally conclude in Section 8

\section{The model}

The Galileon ghost condensate (GGC) model is defined by the following action [28, 31]

$$
\mathcal{S}=\int \mathrm{d}^{4} x \sqrt{-g}\left[\frac{M_{\mathrm{pl}}^{2}}{2} R+a_{1} X+a_{2} X^{2}+3 a_{3} X \square \phi\right],
$$

where $M_{\mathrm{pl}}$ is the Planck mass, $g$ is the determinant of the metric $g_{\mu \nu}, R$ is the Ricci scalar, $X=\nabla_{\mu} \phi \nabla^{\mu} \phi$ with $\phi$ being the scalar field and $\nabla_{\mu}$ the covariant derivative. $a_{1,2,3}$ are constants and $\square \phi=\nabla_{\mu} \nabla^{\mu} \phi$. To the action in (1) we add the matter action $\mathcal{S}_{\mathrm{M}}$, for which we consider perfect fluids minimally coupled to gravity.

Varying the total action with respect to the metric and $\phi$, we obtain the corresponding field equations. We consider for

\footnotetext{
${ }^{1}$ https://www.lsst.org/

2 desi.lbl.gov

3 https://www.euclid-ec.org

4 https://www.skatelescope.org/
}

the background the flat Friedmann-Lemaittre-Robertson-Walker (FLRW) line element, given by

$$
\mathrm{d} s^{2}=-\mathrm{d} t^{2}+a^{2}(t) \gamma_{i j} \mathrm{~d} x^{i} \mathrm{~d} x^{j}
$$

where $a(t)$ is the scale factor and $\gamma_{i j}$ is the spatial metric. Following Ref. [31], we introduce the dimensionless variables

$$
x_{1}=-\frac{a_{1} \dot{\phi}^{2}}{3 M_{\mathrm{pl}}^{2} H^{2}}, \quad x_{2}=\frac{a_{2} \dot{\phi}^{4}}{M_{\mathrm{pl}}^{2} H^{2}}, \quad x_{3}=\frac{6 a_{3} \dot{\phi}^{3}}{M_{\mathrm{pl}}^{2} H},
$$

where $H=\dot{a} / a$, and a dot represents the derivative with respect to the cosmic time $t$. Using these definitions we can write the field equations in the background as a dynamical system:

$$
\begin{array}{ll}
x_{1}^{\prime}=2 x_{1}\left(\epsilon_{\phi}-h\right), & x_{2}^{\prime}=2 x_{2}\left(2 \epsilon_{\phi}-h\right), \\
x_{3}^{\prime}=x_{3}\left(3 \epsilon_{\phi}-h\right), & \Omega_{\mathrm{r}}^{\prime}=-2 \Omega_{\mathrm{r}}(2+h),
\end{array}
$$

where $\Omega_{\mathrm{r}}=\rho_{\mathrm{r}} /\left(3 M_{\mathrm{pl}}^{2} H^{2}\right)$ is the dimensionless density parameter for radiation, $\epsilon_{\phi}=\ddot{\phi} /(H \dot{\phi}), h=\dot{H} / H^{2}$, and a prime is defined as the derivative with respect to $\mathcal{N}=\ln a$. Given the length of the expressions for $\epsilon_{\phi}$ and $h$ we refer the reader to Eqs. (4.16) and (4.17) in Ref. [31] (with $x_{4}=0$ ). From the Friedmann equation we also have

$$
\Omega_{\mathrm{c}}+\Omega_{\mathrm{b}}+\Omega_{\mathrm{r}}+\Omega_{\mathrm{DE}}=1
$$

where $\Omega_{\mathrm{b}, \mathrm{c}}=\rho_{\mathrm{b}, \mathrm{c}} /\left(3 M_{\mathrm{pl}}^{2} H^{2}\right)$ are the density parameter for the baryons (b) and cold dark matter (c), respectively, and

$$
\Omega_{\mathrm{DE}}=x_{1}+x_{2}+x_{3},
$$

is the DE density parameter. Eq. (7), evaluated today, can be used to reduce the number of free parameters of the model, leaving the model with two additional parameters out of three compared to $\Lambda \mathrm{CDM}$, i.e.,

$$
x_{2}^{(0)}=\Omega_{\mathrm{DE}}^{(0)}-x_{1}^{(0)}-x_{3}^{(0)} .
$$

The GGC model allows for a de Sitter fixed point free from ghost instability. The presence of $x_{2} \neq 0$ prevents the model from reaching a tracker solution. The latter would be characterised by $w_{\mathrm{DE}}=-2$ during the matter era, while the $X^{2}$ term allows to temporally enter the region $-2<w_{\mathrm{DE}}<-1$ [31]. This property allows the model to be observationally favoured over $\Lambda \mathrm{CDM}[40]$.

\section{Linear density perturbations}

Let us consider the linear perturbed line element on the flat FLRW background:

$$
\mathrm{d} s^{2}=-(1+2 \Psi) \mathrm{d} t^{2}+a^{2}(t)(1-2 \Phi) \gamma_{i j} \mathrm{~d} x^{i} \mathrm{~d} x^{j},
$$

where $\Psi\left(t, x_{i}\right)$ and $\Phi\left(t, x_{i}\right)$ are the gravitational potentials. In Fourier space, for MG models with one extra scalar dof we can write the following equations which generalise the standard general relativistic Poisson and lensing equations [59.61]:

$$
\begin{aligned}
& -k^{2} \Psi=4 \pi G_{\mathrm{N}} a^{2} \mu^{\mathrm{L}}(a, k) \rho_{\mathrm{m}} \delta_{\mathrm{m}}, \\
& -k^{2}(\Psi+\Phi)=8 \pi G_{\mathrm{N}} a^{2} \Sigma^{\mathrm{L}}(a, k) \rho_{\mathrm{m}} \delta_{\mathrm{m}},
\end{aligned}
$$


where $G_{\mathrm{N}}^{-1}=8 \pi M_{\mathrm{pl}}^{2}$ is the Newtonian gravitational constant, $k$ is the comoving wavenumber, $\rho_{\mathrm{m}} \delta_{\mathrm{m}}=\sum_{i} \rho_{i} \delta_{i}$ is the total matter density perturbation (where $i \in[\mathrm{r}, \mathrm{b}, \mathrm{c}]$ ). The dimensionless quantities $\mu^{\mathrm{L}}$ and $\Sigma^{\mathrm{L}}$ characterise the effective gravitational couplings at linear order felt by matter and light, respectively. The GR limit is recovered when both $\mu^{\mathrm{L}}=\Sigma^{\mathrm{L}}=1$. Applying the quasi-static approximation (QSA) 5 [64, 65] for perturbations inside the scalar field's sound horizon [66] to the model in action (1), it follows that [31]

$$
\mu^{\mathrm{L}}(a)=\Sigma^{\mathrm{L}}(a)=1+\frac{x_{3}^{2}}{Q_{\mathrm{s}} c_{\mathrm{S}}^{2}\left(2-x_{3}\right)^{2}},
$$

where

$$
\begin{aligned}
Q_{\mathrm{s}} & =\frac{3\left(4 x_{1}+8 x_{2}+4 x_{3}+x_{3}^{2}\right)}{\left(2-x_{3}\right)^{2}} \\
c_{\mathrm{s}}^{2} & =\frac{2\left(1+3 \epsilon_{\phi}\right) x_{3}-x_{3}^{2}-4 h-6\left(\Omega_{\mathrm{c}}+\Omega_{\mathrm{b}}\right)-8 \Omega_{\mathrm{r}}}{3\left(4 x_{1}+8 x_{2}+4 x_{3}+x_{3}^{2}\right)} .
\end{aligned}
$$

To avoid ghosts and Laplacian instabilities, we require that both $Q_{\mathrm{s}}$ and the speed of propagation of the scalar modes $c_{\mathrm{s}}^{2}$ are positive. Then, for $x_{3} \neq 0, \mu^{\mathrm{L}}$ and $\Sigma^{\mathrm{L}}$ are larger than 1 . Since $\mu^{\mathrm{L}}=\Sigma^{\mathrm{L}}$, there is no gravitational slip $(\Psi=\Phi)$.

For sub-horizon perturbations, the matter density $\delta_{\mathrm{m}}$ approximately obeys the linear equation

$$
\delta_{\mathrm{m}}^{\prime \prime}+\left(2+\frac{H^{\prime}}{H}\right) \delta_{\mathrm{m}}^{\prime}-\frac{3}{2} \Omega_{\mathrm{m}} \mu^{\mathrm{L}}(a) \delta_{\mathrm{m}}=0,
$$

where we have used Eq. (10) to replace $\Psi$ in favour of $\delta_{\mathrm{m}}$.

We solve the equation above by setting the initial conditions (ICs) as follows: $a_{i}=0.01, \delta_{\mathrm{m}, i}=a_{i}$ and $\delta_{\mathrm{m}, \mathrm{i}}^{\prime}=a_{i}$, which correspond to the matter dominated era solution. The model and cosmological parameters of GGC are listed in Tab. 1 and they correspond to the cosmological constraints obtained with Planck data [40]. For reference we also include the parameters for other two models: the $\Lambda \mathrm{CDM}$ model (for the constraints we refer to [40]) and the Cubic Galileon model (G3) [27]. We decided to use this model for comparison because it can be obtained from GGC by setting $x_{2}=06$ Given this property, the G3 model shows a tracker solution $H^{2} \phi^{\prime}=$ const [67]. The values of the cosmological parameters we use for G3 are from the constraints in Ref. [39]. In this work we decided to use the constrain values of the cosmological/model parameters for each model because we want to make theoretical predictions that are as close as possible to what we can actually expect from observations.

\footnotetext{
${ }^{5}$ In the QSA, time derivatives of the perturbed quantities can be neglected compared with their spatial derivatives. We note that the validity of the QSA for the Horndeski class of models has been proved to be a valid assumption within the scalar field's sound horizon for $k>0.001 \mathrm{~h} / \mathrm{Mpc}$ [62 63]. We have verified that indeed this is the case for the GGC model.

${ }^{6}$ Let us note that the analysis for G3 we show in this work has been the subject of several papers in the past [50,52]. That is why we do not explicitly rewrite the corresponding equations but we prefer to refer the reader to these papers for a detailed discussion.
}

\begin{tabular}{|c|c|c|c|c|c|c|}
\hline Model & $\sigma_{8}^{(0)}$ & $H_{0}$ & $\Omega_{\mathrm{m}}^{(0)}$ & $x_{1}^{(0)}$ & $x_{2}^{(0)}$ & $x_{3}^{(0)}$ \\
\hline$\Lambda$ CDM & 0.83 & 70 & 0.31 & - & - & - \\
\hline G3 & 0.93 & 73.9 & 0.27 & - & - & - \\
\hline GGC & 0.87 & 70 & 0.28 & -1.26 & 1.64 & 0.34 \\
\hline
\end{tabular}

Table 1: Present day values for the amplitude of the linear matter power spectrum at $8 h^{-1} \mathrm{Mpc}, \sigma_{8}^{(0)}$, the Hubble parameter $H_{0}$ in units of $\mathrm{km} \mathrm{s}^{-1} \mathrm{Mpc}^{-1}$, the matter density $\Omega_{m}^{(0)}$ and the $x_{i}^{(0)}$ parameters. They correspond to the maximum likelihood values obtained with Planck data for $\Lambda$ CDM and GGC in Ref. [40], and the mean values of G3 obtained with Planck data in Ref. [39].

In the top panel of Fig. 1, we show the relative difference in the evolution of the linear matter density perturbation $\delta_{\mathrm{m}}$ with respect to $\Lambda \mathrm{CDM}$ for both the GGC and the G3 models. The relative difference is very small at early times for both models. In the case of GGC, it remains smaller than $1 \%$ throughout its growth history, while for G3 it reaches $11 \%$ at present. Such modifications with respect to the $\Lambda \mathrm{CDM}$ model are due in both cases to a modified expansion history and to $\mu^{\mathrm{L}} \neq 1$ at later times, while the large difference between the GGC and the G3 is only due to the presence of $x_{2} \neq 0$ in GGC.

Modifications with respect to $\Lambda \mathrm{CDM}$ can be also spotted in the linear growth rate $f(a)$, which is a derived quantity defined as

$$
f(a)=\frac{\mathrm{d} \ln \delta_{\mathrm{m}}}{\mathrm{d} \ln a} .
$$

We show its evolution in the bottom panel of Fig. 1 for the two Galileon models and we compare them to $\Lambda$ CDM. The growth rates in both Galileon models become larger than $\Lambda \mathrm{CDM}$ as soon as the Universe exits the matter dominated era. Appreciable differences in the case of GGC are around $a=0.2$, being the time at which $\delta_{\mathrm{m}}(\mathrm{GGC})$ starts to be larger than that of $\Lambda \mathrm{CDM}$. For GGC, the linear growth rate $f$ is enhanced with respect to the standard model until $a \gtrsim 0.6$, while at earlier times the difference is negligible. In G3 differences arise a bit earlier because a $0.5 \%$ difference in $\delta_{\mathrm{m}}$ is already present (see upper panel). A large enhanced modification is then present up to the present time.

\section{Non-linear perturbations}

We will now investigate the evolution of the metric and the scalar field perturbations on small scales, where second order, non-linear perturbations are no longer negligible. Let us consider the perturbation of the scalar field: $\phi\left(t, x_{i}\right)=\phi(t)+\delta \phi\left(t, x_{i}\right)$ and along with the QSA we will also neglect terms that are suppressed by the Newtonian potentials and their first spatial derivatives.

Then, the time-time component of the GGC equation gives

$$
\frac{\partial^{2} \Phi}{a^{2}}=4 \pi G_{\mathrm{N}} \rho_{\mathrm{m}} \delta_{\mathrm{m}}+24 \pi G_{\mathrm{N}} a_{3} X \frac{\partial^{2} \delta \phi}{a^{2}}
$$

where the derivatives are with respect to spatial components, 

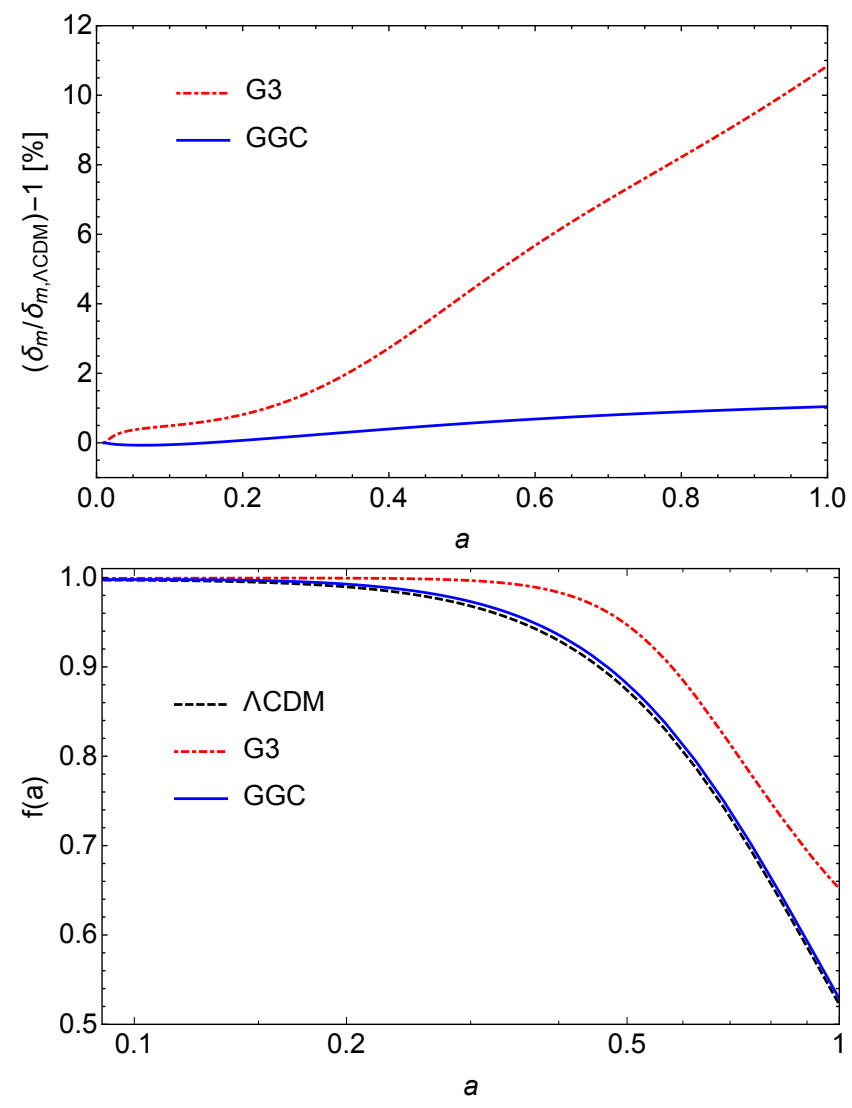

Figure 1: Top panel: Time evolution of the relative difference in percentage of the linear matter density perturbation in the GGC (blue solid line), G3 (red dot-dashed line) with respect to the $\Lambda$ CDM. Bottom panel: Evolution of the linear growth rate for GGC, G3 and $\Lambda$ CDM (black dashed line). The model and cosmological parameters are shown in Tab. 1 .

and the equation for the scalar field reads

$$
\begin{aligned}
-3 a_{3} \dot{\phi}^{2} \frac{\partial^{2} \Psi}{a^{2}} & =\left[-a_{1}-2 a_{2} X+6 a_{3}(\ddot{\phi}+2 H \dot{\phi})\right] \frac{\partial^{2} \delta \phi}{a^{2}} \\
& +3 a_{3}\left[\left(\frac{\partial^{2} \delta \phi}{a^{2}}\right)^{2}-\left(\frac{\partial_{i} \partial_{j} \delta \phi}{a^{2}}\right)^{2}\right]
\end{aligned}
$$

where $\left(\partial_{i} \partial_{j} \delta \phi\right)^{2}=\left(\partial_{i} \partial_{j} \delta \phi\right)\left(\partial^{i} \partial^{j} \delta \phi\right)$ and indexes are raised with the metric $\gamma_{i j}: \partial^{i}=\gamma^{i j} \partial_{j}$.

At the non-linear level, the relation $\Phi=\Psi$ is still valid, so we can combine the above equations to get

$$
\frac{\partial^{2} \delta \phi}{a^{2}}+\lambda^{2}\left[\left(\frac{\partial^{2} \delta \phi}{a^{2}}\right)^{2}-\left(\frac{\partial_{i} \partial_{j} \delta \phi}{a^{2}}\right)^{2}\right]=-4 \pi G_{\mathrm{N}} \zeta \rho_{\mathrm{m}} \delta_{\mathrm{m}},
$$

where

$$
\lambda^{2}(a)=\frac{12 a_{3} \dot{\phi}^{2}}{M_{\mathrm{pl}}^{2} H^{2} c_{\mathrm{s}}^{2} Q_{\mathrm{s}}\left(2-x_{3}\right)^{2}}, \quad \zeta(a)=\lambda^{2} \dot{\phi}^{2} .
$$

Let us consider a spherically symmetric density perturbation. Then, Eq. (19) becomes

$$
\frac{1}{r^{2}} \frac{\mathrm{d}}{\mathrm{d} r}\left(r^{2} \frac{\mathrm{d} \delta \phi}{\mathrm{d} r}\right)-\frac{2 \lambda^{2}}{r^{2}} \frac{\mathrm{d}}{\mathrm{d} r}\left[r\left(\frac{\mathrm{d} \delta \phi}{\mathrm{d} r}\right)^{2}\right]=-4 \pi G_{\mathrm{N}} \zeta \rho_{\mathrm{m}} \delta_{\mathrm{m}}
$$

Defining the mass enclosed in a sphere of radius $r$ as

$$
m(r)=4 \pi \int_{0}^{r} r^{\prime 2} \rho_{\mathrm{m}}\left(r^{\prime}\right) \delta_{\mathrm{m}}\left(r^{\prime}\right) \mathrm{d} r^{\prime},
$$

we can integrate Eq. (19) and obtain

$$
r^{2} \frac{\mathrm{d} \delta \phi}{\mathrm{d} r}-2 \lambda^{2} r\left(\frac{\mathrm{d} \delta \phi}{\mathrm{d} r}\right)^{2}=-G_{\mathrm{N}} \zeta m(r)
$$

We can now evaluate its solution, which reads

$$
\frac{\mathrm{d} \delta \phi}{\mathrm{d} r}=\frac{r_{\mathrm{V}}}{4 \lambda^{2}}\left[\frac{r}{r_{\mathrm{V}}}\left(1-\sqrt{1+\frac{r_{\mathrm{V}}^{3}}{r^{3}}}\right)\right],
$$

where $r_{\mathrm{V}}$ is the Vainshtein radius of the enclosed mass perturbation and it is defined as

$$
r_{\mathrm{V}}^{3}=8 G_{\mathrm{N}} m(r) \lambda^{2} \zeta=\frac{32 G_{\mathrm{N}} m(r) x_{3}^{2}}{\left[H c_{\mathrm{S}}^{2} Q_{\mathrm{s}}\left(2-x_{3}\right)^{2}\right]^{2}} .
$$

It then depends on the mass distribution in the sphere and on the parameters of the model. In particular, it is non-vanishing as long as $x_{3} \neq 0$. For a point source, $r_{\mathrm{V}}=2.07 \times 10^{2}\left(M / M_{\odot}\right)^{1 / 3}$ $\mathrm{pc}$, where we have used the maximum likelihood values for the present day parameters $H_{0}, x_{3}, x_{2}, x_{1}$ obtained in [40] with Planck data. The corresponding Vainshtein radius for G3 is $r_{\mathrm{V}}=2.24 \times 10^{2}\left(M / M_{\odot}\right)^{1 / 3}$ pc where we have used the constraints obtained in [39] (see Tab. 1). We note that the Vainshtein radius at the present time for the GGC is smaller than the one for the G3. In Fig. 2 we compare the time evolution of the Vainshtein radius for both the GGC and the G3 models. For both models, at early times, its value is very small which means that the screening mechanism works only on very small scales. At this time the Vainshtein radius for the GGC is slightly larger than the G3 one and afterwards they become equal. As the Universe expands, the GGC radius increases and its value is higher if compared to G3 in the time range $0.07<a<0.9$. Only at present time we notice a change of trend, the Vainshtein radius of the GGC decreases with respect to the G3 according to the estimated values we presented before.

According to Eq. 24, well outside the Vainshtein radius, the derivative of the scalar field perturbation is proportional to the Newtonian potential and it corresponds to the linear solution.

If we consider a top-hat profile for the density field, we get that $\mathrm{d} \delta \phi / \mathrm{d} r \propto r$ for $r<R$ where $R$ is the radius of the sphere of mass $m(R)=M$. Then, Eq. (19) reduces to

$$
\frac{\partial^{2} \delta \phi}{a^{2}}-\frac{2 \lambda^{2}}{3}\left(\frac{\partial^{2} \delta \phi}{a^{2}}\right)^{2}=-4 \pi G_{\mathrm{N}} \zeta \rho_{\mathrm{m}} \delta_{\mathrm{m}}
$$

At $r=R$ the equation above can be solved for $\partial^{2} \delta \phi / a^{2}$ and we find

$$
\frac{\partial^{2} \delta \phi}{a^{2}}=8 \pi G_{\mathrm{N}} \rho_{\mathrm{m}} \zeta\left(\frac{R}{R_{\mathrm{V}}}\right)^{3}\left[1-\sqrt{1+\frac{R_{\mathrm{V}}^{3}}{R^{3}}}\right] \delta_{\mathrm{m}}
$$




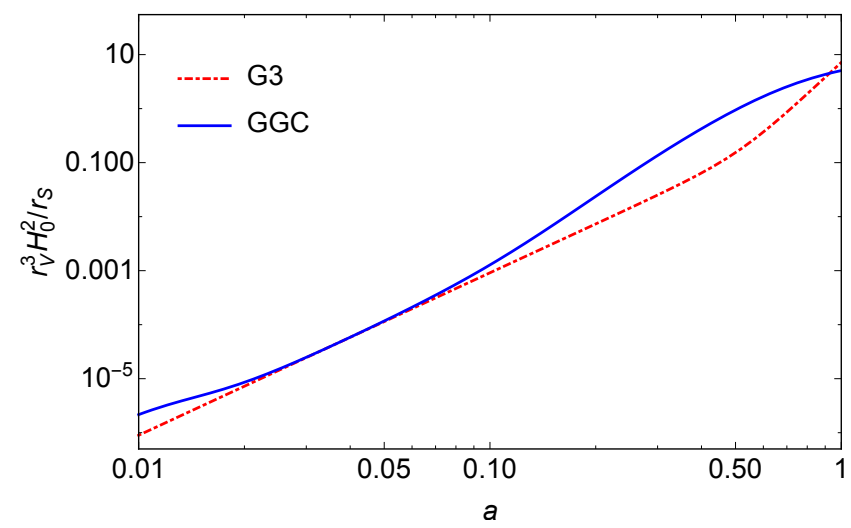

Figure 2: Time evolution of the Vainshtein radius for the GGC (blue solid line) and G3 (red dot-dashed line) models. We have defined $r_{\mathrm{s}}=2 G_{\mathrm{N}} M$ as the Schwarzschild radius. The parameters for the GGC and the G3 models have been chosen according to the cosmological constraints in [40] and [39], respectively.

where $R_{\mathrm{V}}^{3}=8 G_{\mathrm{N}} \lambda^{2} \zeta \delta M$ and $\delta M$ is the total mass of the density perturbation $\rho_{\mathrm{m}} \delta_{\mathrm{m}}$. Now we can compute a modified Poisson equation from Eq. 17) which includes non-linear corrections and it reads

$$
\frac{\partial^{2} \Psi}{a^{2}}=4 \pi G_{\mathrm{N}} \mu^{\mathrm{NL}}(a, R) \rho_{\mathrm{m}} \delta_{\mathrm{m}},
$$

where the non-linear effective gravitational coupling is

$$
\mu^{\mathrm{NL}}(a, R)=1+2\left(\mu^{\mathrm{L}}-1\right)\left(\frac{R}{R_{\mathrm{V}}}\right)^{3}\left(\sqrt{1+\frac{R_{\mathrm{V}}^{3}}{R^{3}}}-1\right) .
$$

In the limit $R \rightarrow 0$ the above expression reduces to unity approaching GR, while for $R \gg R_{\mathrm{V}}$ we recover the linear result $\mu^{\mathrm{NL}} \rightarrow \mu^{\mathrm{L}}$, showing how the Vainshtein screening mechanism works.

In the next Section we will show the evolution of $\mu^{\mathrm{NL}}$ for a collapsing sphere and we will compare it with the linear effective gravitational coupling. Finally, because in the non-linear regime $\Phi=\Psi$ is still valid, we can deduce $\Sigma^{\mathrm{NL}}=\mu^{\mathrm{NL}}$. This information will be used when computing the non-linear lensing power spectrum.

\section{Spherical collapse model}

The spherical collapse process is the simplest model for the formation of non-linear gravitationally bound structures. It is characterised by the turnaround phase, during which the amplitude of the spherical perturbation in the expansion phase reaches a sufficient large value such that the gravitational force prevents the sphere from an infinite expansion. It is then followed by the proper collapse phase, i.e., when the sphere reaches its maximum radius at the turnaround, $R_{\mathrm{ta}}$, the overdensity starts to collapse. While the mathematical model implies the collapsing sphere to reduce to a point, in reality this does not happen, as virialization takes place [68, 69] and the system satisfies the Virial theorem. In the following we assume that during the evolution the matter distribution remains with a top-hat profile.
The non-linear evolution equation for the matter overdensity is [70, 71]

$$
\ddot{\delta}_{\mathrm{m}}+2 H \dot{\delta}_{\mathrm{m}}-\frac{4}{3} \frac{\dot{\delta}_{\mathrm{m}}^{2}}{1+\delta_{\mathrm{m}}}=\left(1+\delta_{\mathrm{m}}\right) \frac{\partial^{2} \Psi}{a^{2}} .
$$

We can use Eq. 28) to eliminate the metric potential. Thus it is clear that the evolution of $\delta_{\mathrm{m}}$ is modified with respect to GR by $\mu^{\mathrm{NL}}$.

Assuming that the total mass inside $R$ is conserved during the collapse, we have

$$
M=\frac{4 \pi}{3} R^{3} \rho_{\mathrm{m}}\left(1+\delta_{\mathrm{m}}\right)=\text { const },
$$

from which we can derive the equation of the evolution of the radius after differentiating it with respect to time and using Eq. 30. Then, we have

$$
\frac{\ddot{R}}{R}=H^{2}+\dot{H}-\frac{4 \pi G_{\mathrm{N}}}{3} \mu^{\mathrm{NL}} \rho_{\mathrm{m}} \delta_{\mathrm{m}},
$$

which is composed by a background term $\left(H^{2}+\dot{H}\right)$ and a gravitational one $\left(\propto \mu^{\mathrm{NL}} \rho_{\mathrm{m}} \delta_{\mathrm{m}}\right)$. We can numerically solve the above equation as follows. As standard procedure, we introduce the variable

$$
y=\frac{R}{R_{i}}-\frac{a}{a_{i}}
$$

where $R_{i}$ is the initial radius of the perturbation and $a_{i}$ is the initial scale factor. Thus Eq. 32, reads

$$
y^{\prime \prime}=-\frac{H^{\prime}}{H} y^{\prime}+\left(1+\frac{H^{\prime}}{H}\right) y-\frac{\Omega_{\mathrm{m}}}{2} \mu^{\mathrm{NL}} \delta_{\mathrm{m}}\left(y-\frac{a}{a_{i}}\right) .
$$

In order to specify the evolution of $\mu^{\mathrm{NL}}(a, R)$, we also use

$$
\left(\frac{R}{R_{\mathrm{V}}}\right)^{3}=\frac{1}{4 \Omega_{\mathrm{m}} H^{2} \lambda^{2} \zeta} \frac{1}{\delta_{\mathrm{m}}}=\frac{x_{3}^{2}}{16 \Omega_{\mathrm{m}}\left(\mu^{\mathrm{L}}-1\right)^{2}} \frac{1}{\delta_{\mathrm{m}}},
$$

which can be easily computed from the mass conservation and the definition of Vainshtein radius. Eq. (35) thus shows the relation between the Vainshtein radius and the collapsing overdensity, which holds as long as $x_{3} \neq 0$.

In order to solve Eq. (34) numerically, we consider the initial conditions such that the collapse time is $a_{\text {collapse }}=1$. It follows $a_{i}=6.66 \times 10^{-6}, y_{i}=0$ and $y_{i}^{\prime}=-\delta_{\mathrm{m}, i} / 3$, where $\delta_{\mathrm{m}, i}$ is the initial density obtained from linear theory in the matter dominated era assuming the collapse $(R=0)$ at $a_{\text {collapse }}=1$. Finally, we write the overdensity as

$$
\delta_{\mathrm{m}}=\left(1+\delta_{\mathrm{m}, i}\right)\left(1+\frac{a_{i}}{a} y\right)^{-3}-1,
$$

which follows from matter conservation.

In Fig. 3 we show the solution of Eq. (32) for the three models when the collapse time is set at the present time. We note

\footnotetext{
${ }^{7}$ From Eq. $\sqrt{33}$ at initial time one gets $y_{i}=0$ and $y_{i}^{\prime}=-\delta_{\mathrm{m}, i}^{\prime} /\left(3\left(1+\delta_{\mathrm{m}, i}\right)\right)$. Assuming that the density perturbation grows linearly during matter dominated era, we can use $\delta_{\mathrm{m}} \propto a$ and $\delta_{\mathrm{m}}^{\prime} \propto \delta_{\mathrm{m}}$, thus one gets $y_{i}^{\prime}=-\delta_{\mathrm{m}, i} / 3$ (see Ref. [51]).
} 


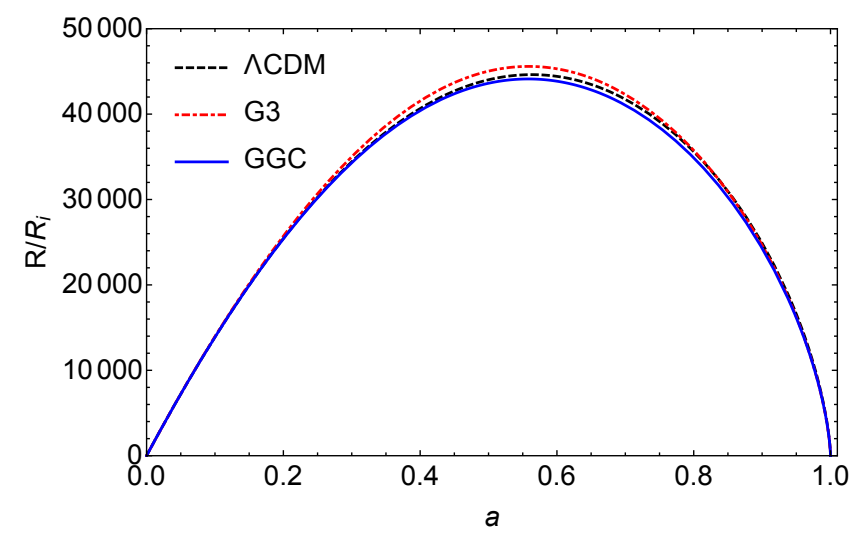

Figure 3: Time evolution of $R / R_{i}$ for the GGC (blue solid line) and G3 (red dotdashed line) and $\Lambda$ CDM (black dashed line) models. The initial overdensity $\delta_{\mathrm{m}, i}$ for the models are: $\delta_{\mathrm{m}, i}(\Lambda \mathrm{CDM})=12.3 \times 10^{-5}, \delta_{\mathrm{m}, i}(\mathrm{GGC})=13.2 \times 10^{-5}$ and $\delta_{\mathrm{m}, i}(\mathrm{G} 3)=7.3 \times 10^{-5}$. The model and cosmological parameters are shown in Tab. 1

\begin{tabular}{|c|c|c|c|}
\hline & $\Lambda$ CDM & G3 & GGC \\
\hline$\delta_{\mathrm{c}}$ & 1.675 & 1.738 & 1.708 \\
\hline$\Delta_{\text {vir }}$ & 333.1 & 317.6 & 342.8 \\
\hline$a_{\mathrm{ta}}$ & 0.563 & 0.558 & 0.558 \\
\hline$a_{\mathrm{vir}}$ & 0.922 & 0.916 & 0.919 \\
\hline$R_{\mathrm{ta}} / R_{i}$ & 44621.5 & 45583.9 & 44114.4 \\
\hline$R_{\mathrm{vir}} / R_{i}$ & 21639.3 & 22285.0 & 21434.5 \\
\hline
\end{tabular}

Table 2: Physical quantities characterising the spherical collapse at the present time for the GGC model in comparison with $\Lambda$ CDM and G3. The parameters of the models used to obtain these results are in Tab. 1

that modifications with respect to the $\Lambda \mathrm{CDM}$ model are present during the collapse phase for both Galileon models. However, the modification introduced by $x_{2}$ makes the dynamics of the collapse for the GGC quite different from that of the G3. The $\Lambda C D M$ model indeed is in between the two Galileon models. This is understood by noticing that there is the following hierarchy for the initial overdensities: $\delta_{\mathrm{m}, i}(\mathrm{G} 3)<\delta_{\mathrm{m}, i}(\Lambda \mathrm{CDM})<$ $\delta_{\mathrm{m}, i}(\mathrm{GGC})$. This translates to an opposite hierarchy for the radii, as a larger overdensity implies an earlier collapse and therefore a smaller radius. The GGC model has a turn-around radius smaller than both the $\Lambda \mathrm{CDM}$ and the G3 as shown in Tab. 2 The latter, instead, shows the larger one. The turn-around phase takes place at the same time for both the Galileon cosmologies, $a_{\mathrm{ta}} \approx 0.558$ while in $\Lambda \mathrm{CDM}$ it is slightly delayed, $a_{\mathrm{ta}} \approx 0.567$.

In Fig. 4, we show the time evolution of the non-linear effective gravitational coupling compared to the linear one for both the GGC and the G3 models. We note that the matter overdensity for the GGC case enters the Vainshtein radius approaching the GR solution before the G3 model does. The crossing time of the Vainshtein radius can be extrapolated from Fig. 5 where we show the evolution of $\left(R / R_{\mathrm{V}}\right)^{3}$. It occurs when $R / R_{\mathrm{V}}=1$ and for the GGC it is at $a=0.24$ and for the G3 at $a=0.47$.

The final stage of the collapse is virialization. The collapse stops when the system reaches the equilibrium and thus satisfies the Virial theorem. The latter states that, for a stable, self-

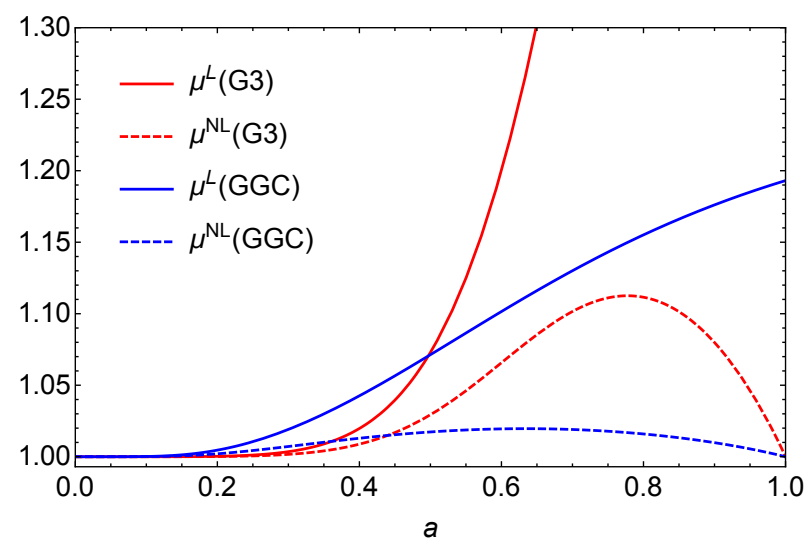

Figure 4: Time evolution of $\mu^{\mathrm{L}}$ (solid lines) and $\mu^{\mathrm{NL}}$ (dashed lines) for the GGC (blue) and the G3 (red) models. The models' parameters for the GGC and the G3 have been chosen according to the cosmological constraints in Refs. [40] and [39], respectively.

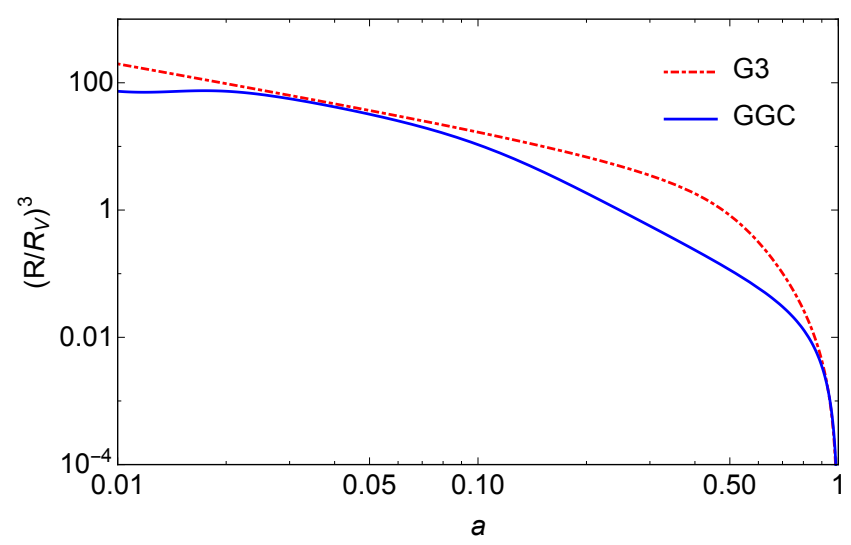

Figure 5: Time evolution of $R / R_{\mathrm{V}}$ for the GGC (blue solid line) and the G3 (red dot-dashed line) models for a matter overdensity collapsing at the present time. The models' parameters for the GGC and the G3 have been chosen according to the cosmological constraints in Refs. [40] and [39], respectively.

gravitating, spherical distribution, the total kinetic energy of the object $(T)$ and the total gravitational potential energy $(U)$, satisfy the relation:

$$
T+\frac{1}{2} U=0
$$

where the kinetic energy during the collapse for a top-hat profile is

$$
T \equiv \frac{1}{2} \int \mathrm{d}^{3} x \rho_{\mathrm{m}} \mathrm{v}^{2}=\frac{3}{10} M \dot{R}^{2},
$$

and the total potential energy is [50]

$$
\begin{aligned}
U & \equiv-\int \mathrm{d}^{3} x \rho_{\mathrm{m}}(\mathbf{x}) \mathbf{x} \cdot \nabla \Psi \\
& =\frac{3}{5}\left(\dot{H}+H^{2}\right) M R^{2}-\frac{3}{5} G_{\mathrm{N}} \mu^{\mathrm{NL}} \frac{M}{R} \delta M .
\end{aligned}
$$

Let us note that the energy conservation is not strictly satisfied for a time-dependent dark energy or modified gravity model [49]. Thus, we choose the virialization time $a_{\text {vir }}$ such that the conservation relation (37) is satisfied. We can then define the 
virial overdensity as

$$
\Delta_{\text {vir }} \equiv \frac{\rho_{\text {vir }}}{\rho_{\text {collapse }}}=\left[1+\delta_{\mathrm{m}}\left(R_{\text {vir }}\right)\right]\left(\frac{a_{\text {collapse }}}{a_{\text {vir }}}\right)^{3} .
$$

In Tab. 1 we list some relevant physical quantities such as $a_{\text {vir }}, R_{\text {vir }} / R_{i}, \Delta_{\text {vir }}$ and $\delta_{\mathrm{c}}$ for a matter overdensity collapsing at the present time. $\delta_{\mathrm{c}}$ is the linear critical density contrast defined as the value of the linear $\delta_{\mathrm{m}}$ at the collapse when initial conditions are assumed such that the non-linear equation diverges at the collapse time. Knowing the initial overdensity $\delta_{\mathrm{i}}$ and its time derivative $\dot{\delta}_{\text {i }}$ leading to collapse at a given time, one solves the linearised version of Eq. (30) to obtain the linear critical overdensity $\delta_{\mathrm{c}}$. This is mathematically equivalent to solve Eq. (15) and to rescale $\delta_{\mathrm{i}}$ by the linear growth factor of the corresponding cosmological model.

In Figs. 6 and 7 we show the evolution of $\delta_{c}$ and $\Delta_{\text {vir }}$ respectively as function of the scale factor. The critical density at early times approaches the value of the Einstein-de Sitter Universe, and for $a>0.2$ its time evolution differs in the three cosmological models, as the contribution of the cosmological constant and of the modifications of gravity become more important with time. In detail, while $\delta_{\mathrm{c}}(\Lambda \mathrm{CDM})$ decreases approaching the collapse at $a=1$, in the Galileon cosmologies the late time values of $\delta_{\mathrm{c}}$ are larger: $\delta_{\mathrm{c}}(\mathrm{GGC})$ increases up to 1.708 while $\delta_{\mathrm{c}}(\mathrm{G} 3)$ is rather constant till $a \approx 0.4$ and then it rapidly grows up to 1.738 . From Fig. 7 we see that the evolution of the virial overdensity for the $\Lambda \mathrm{CDM}$ and the GGC is approximately the same and $\Delta_{\text {vir }}$ (GGC) prefers slightly larger values for $a>0.5 . \Delta_{\text {vir }}(\mathrm{G} 3)$ remains constant $(\approx 177.8$, the Einstein-de Sitter value) up to $a \approx 0.4$ and then increases remaining always smaller than $\Lambda \mathrm{CDM}$ and GGC.

We note that both the GGC and the G3 share in the Lagrangian the same form for the cubic term $(\propto X \square \phi)$, but in the latter the ghost condensate term $\propto X^{2}$ is not present. Although the modifications at both linear and non-linear regimes are driven by the cubic term, the inclusion of the $X^{2}$ term changes the evolution of the scalar field and that of the background quantities in such a way that all the relevant physical quantities we have investigated in this section show a significant modification with respect to the G3 model. Finally, we recall that these results are obtained using the best fit values for the parameters of each model from cosmological data on linear scales, thus according to data, the theoretical predictions we obtain are very close to what we can actually expect at non-linear level.

\section{Non-linear matter and lensing power spectra}

Matter and lensing auto-correlation power spectra are two powerful tools to investigate the deviations from GR. One can resort to Einstein-Boltzmann codes to compute their predictions at linear scales [72] (see Ref. [40] for GGC). In order to extend such predictions on smaller scales one has to include non-linear corrections and screening mechanisms effects. These usually require a model by model implementation of the relevant equations in $N$-body codes [52, 73, 87].

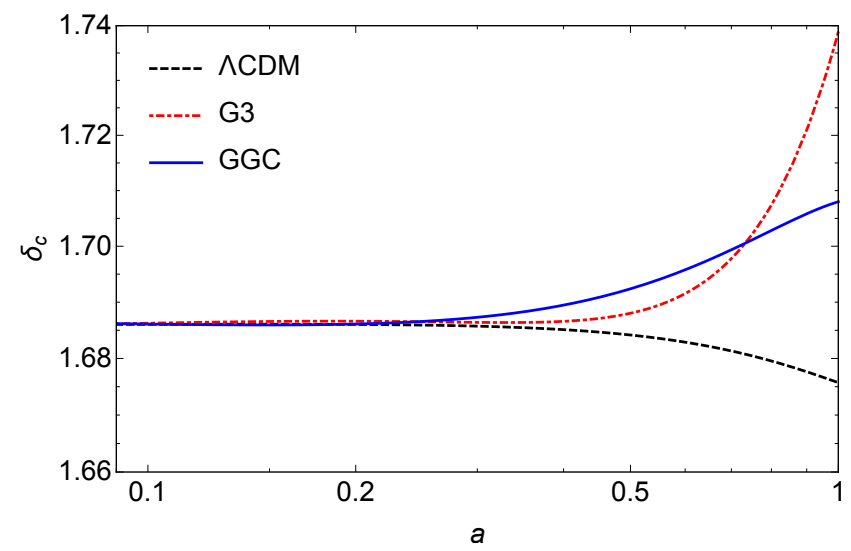

Figure 6: Time evolution of $\delta_{\mathrm{c}}$ as function of the scale factor for the three cosmological models: $\Lambda$ CDM (black dashed line), GGC (blue solid line) and G3 (red dot-dashed line).

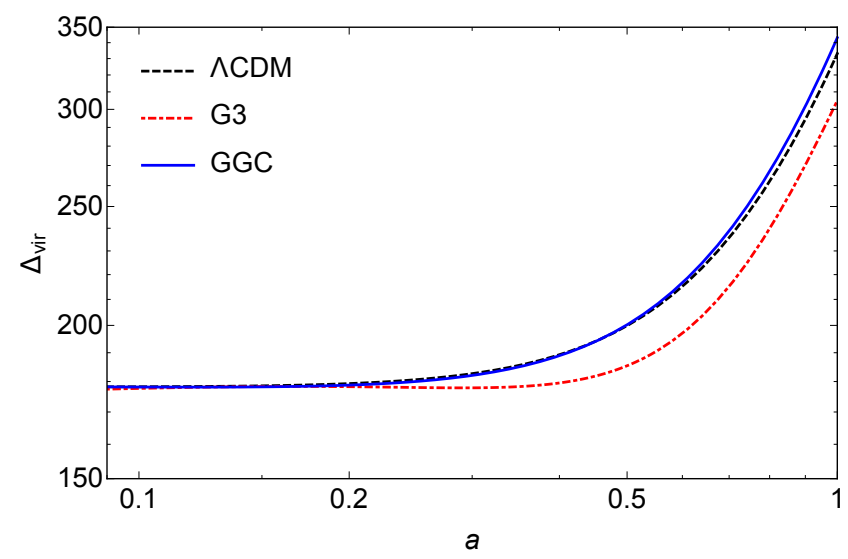

Figure 7: Time evolution of $\Delta_{\mathrm{vir}}$ as a function of the scale factor for $\Lambda \mathrm{CDM}$ (black dashed line), GGC (blue solid line) and G3 (red-dot dashed line).

Analytically, a formalism to calculate the non-linear matter power spectrum for wider classes of gravity models has been developed [88] considering the closure approximation [89] with applications to DGP and $f(R)$ gravity models; or another approach [90] is the one which extends the reaction method [91] using the halo model. Alternatively, a parameterization based on spherical collapse computations capturing the non-linear MG effects on structure formation has been recently proposed and implemented in an $N$-body code [92].

In this work the goal is to have a glimpse into the phenomenology associated with the screening effects on the matter and lensing power spectra, leaving for a future work a more detailed investigation. In this regard, we will use the predictions from linear cosmological perturbation theory and incorporate the screening effects in a phenomenological fashion [93 95]. We will model the small-scale limit to GR through a direct dependence on the screening scale in the matter power spectrum, as follows: 


$$
\begin{aligned}
P_{G G C}^{\mathrm{NL}}(k, z) & =\frac{P_{\Lambda C D M}^{\mathrm{NL}}(k, z)}{P_{\Lambda C D M}^{\mathrm{L}}(k, z)}\left\{P_{\Lambda C D M}^{\mathrm{L}}(k, z)\right. \\
& \left.+\left[P_{G G C}^{\mathrm{L}}(k, z)-P_{\Lambda C D M}^{\mathrm{L}}(k, z)\right] e^{-\left(\frac{k}{k_{s}}\right)^{2}}\right\}
\end{aligned}
$$

where $k_{\mathrm{s}}$ is the screening scale. The linear power spectrum of $\Lambda \mathrm{CDM}$ and GGC are respectively obtained from the EinsteinBoltzmann solver CAMB [96] and EFTCAMB [97, 98]. The non-linear matter power spectrum for $\Lambda \mathrm{CDM}$ is obtained using the prescription in Ref. [99]. The cosmological parameters are the same for $\Lambda \mathrm{CDM}$ and GGC in Eq. (41), in particular they are those of GGC. The Eq. 41 recovers $P_{G G C}^{L}(k, z)$ in the limit $k \ll k_{\mathrm{s}}$ and $P_{\Lambda C D M}^{\mathrm{NL}}(k, z)$ in the regime $k \gg k_{\mathrm{s}}$.

The value of the screening scale is strictly related to the specific model under consideration. From $N$-body cosmological simulations in the G3 model one finds that $k_{\mathrm{s}}=0.1 \mathrm{h \textrm {Mpc } ^ { - 1 }}$ at the present time [52]. For the GGC model, $N$-body simulations do not exist, therefore we will present our results for four values of $k_{\mathrm{s}}$ in order to quantify the relevance of this parameter. They are $k_{\mathrm{s}}=0.05,0.1,0.5,1 \mathrm{~h} \mathrm{Mpc}^{-1}$ and will serve to show the phenomenology of GGC at these scales and provide theoretical predictions to be then compared to accurate $N$-body simulations once they are available. We guess that the more reliable results for the GGC will be those with a screening scale larger than that of the G3 at the present time. That is because we find that the Vainshtein radius at the present time for a point source for GGC is slightly smaller than that in the G3 model, therefore we expect $k_{\mathrm{s}}(\mathrm{GGC})>k_{\mathrm{s}}(\mathrm{G} 3)$ at $z=0$. Note, though, that $k_{\mathrm{s}}(\mathrm{GGC}) \gtrsim k_{\mathrm{s}}(\mathrm{G} 3)$ only very recently, while for the majority of the cosmic history, $k_{\mathrm{S}}(\mathrm{GGC}) \lesssim k_{\mathrm{s}}(\mathrm{G} 3)$, as it can be easily seen from the evolution of the Vainshtein radius in Figs. 2 and 5 However, determining the time evolution of $k_{\mathrm{s}}$ is not an easy task and it is necessary to use $N$-body simulations for its accurate determination. Its time dependence is further confirmed in the case of the G3 model in Ref. [52] using $N$-body simulations. For the $\mathrm{G} 3$ model the screening scale is $k_{\mathrm{s}} \simeq 0.1 \mathrm{h \textrm {Mpc } ^ { - 1 }}$ at $a=1$ and $k_{\mathrm{s}} \simeq 0.3-0.4 h \mathrm{Mpc}^{-1}$ at $a=0.6$. This is a clear indication that a constant $k_{\mathrm{s}}$ might just be a first approximation. Therefore to avoid introducing further phenomenological approaches, we prefer to consider the screening scale $k_{\mathrm{s}}$ constant in time, in agreement with current literature on the subject [93-95]. While this approach does have a marginal effect on the study of the matter power spectrum, it might have relevance in the computation of the lensing power spectrum, as it requires the knowledge of the time evolution of both the matter power spectrum and the screening scale.

In Fig. 8 we present the non-linear matter power spectrum at $z=0$ for $\Lambda \mathrm{CDM}$ and the GGC model for the four screening scales discussed above and the relative difference $\Delta P / P$ in the bottom panel, where $\Delta P=P^{\mathrm{GGC}}(k)-P^{\Lambda \mathrm{CDM}}(k)$ and $P=P^{\Lambda \mathrm{CDM}}$. The top figure compares the two models having their best-fit parameters, while the bottom figure compares the GGC with the $\Lambda \mathrm{CDM}$ having the same cosmological parameters of the GGC. When comparing the two models with their best-fit parameters, on large scales, $k \lesssim 10^{-3} h \mathrm{Mpc}^{-1}$, the
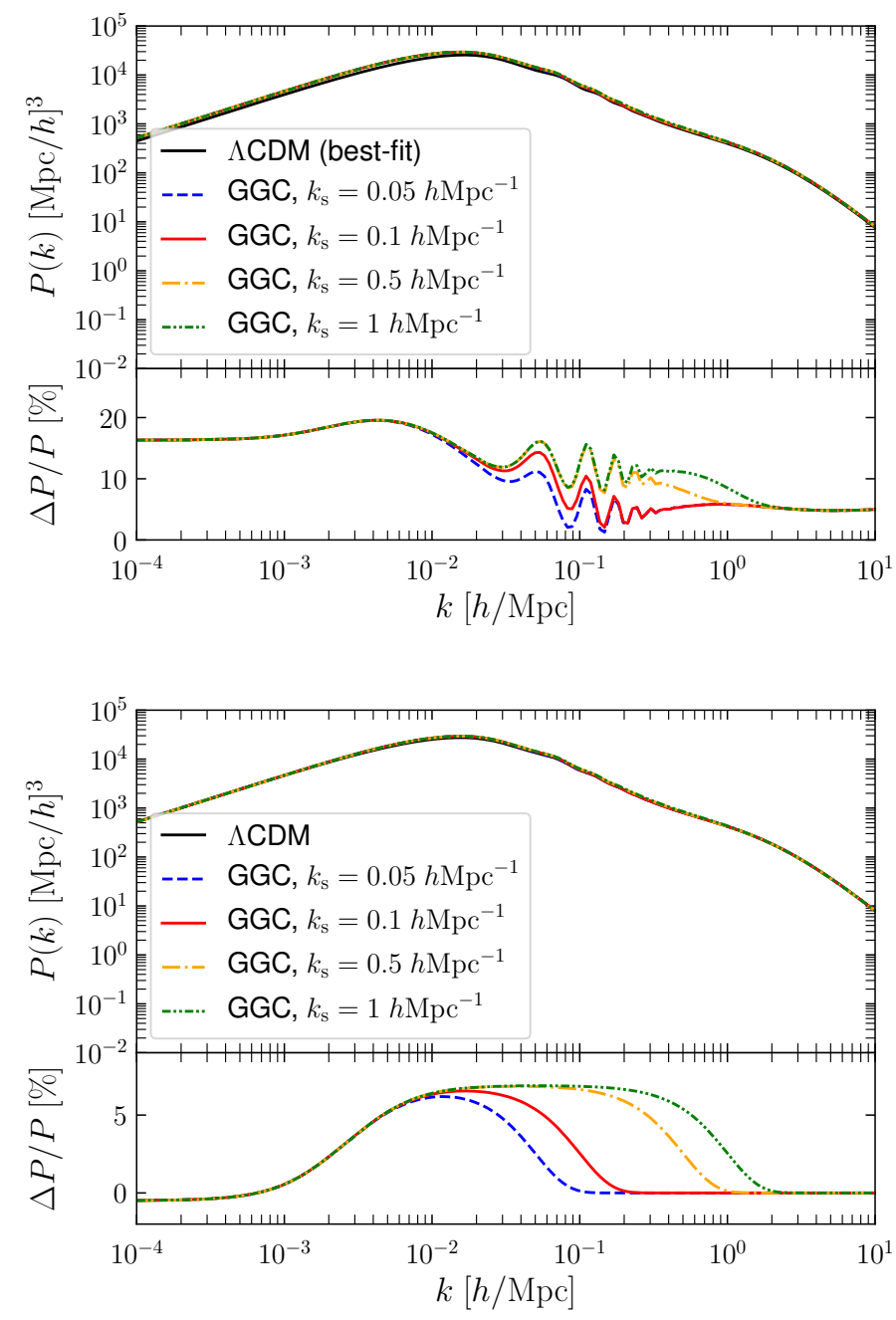

Figure 8: Non-linear matter power spectra as a function of $k$ at $z=0$ of the $\Lambda \mathrm{CDM}$ model (solid black line) and of the GGC model, and non-linear matter power spectra percentage relative difference of the GGC model with respect to $\Lambda \mathrm{CDM}$. For the GGC we show the results for different screening scales $k_{\mathrm{s}}$ : $0.05 h \mathrm{Mpc}^{-1}$ (dashed blue line), $0.1 h \mathrm{Mpc}^{-1}$ (solid red line), $0.5 h \mathrm{Mpc}^{-1}$ (dotdashed orange line) and $1 \mathrm{~h} \mathrm{Mpc}^{-1}$ (dashed-dot-dotted green line). The top figure compares the $\Lambda \mathrm{CDM}$ and the GGC models with the respective best-fit parameters (Tab. 1), while the bottom one compares the GCC model with $\Lambda \mathrm{CDM}$ having the same cosmological parameters of GGC.

difference is smaller than $20 \%$ between the two models due to the different normalizations of the spectra and a different behaviour of linear perturbations. The difference slightly increases on intermediate scales, up to $20 \%$ and then decreases to approximately $5 \%$ on small scales where the Vainshtein screening takes place. The exact scale depends on the value of the screening scale, $k_{\mathrm{s}}$. A small value of the latter induces a suppression of power on larger scales (small $k$ ) with respect to a larger value of $k_{\mathrm{s}}$. This is indeed evident when comparing the results for $k_{\mathrm{s}}=0.1 \mathrm{~h} \mathrm{Mpc}^{-1}$ and $k_{\mathrm{s}}=0.5 \mathrm{~h} \mathrm{Mpc}^{-1}$ : a factor of 5 in the screening scale translates into about a factor of two in the scale where one would approximately recover the $\Lambda \mathrm{CDM}$ limit. The main difference in changing the screening scale is given by the scale at which the screening starts to be important. 
After reaching the maximum, for small values of the screening scale, the model looses power relatively fast, while large values of the screening scale lead to a slower decline of the power. All the GGC models, by construction, lead to the same plateau, which differs from zero as the GGC and $\Lambda \mathrm{CDM}$ models do not share the same cosmological parameters and as such it is not expected that the $\Lambda \mathrm{CDM}$ limit of the GGC power spectrum at non-linear scale coincides with that of $\Lambda C D M$ with best-fit parameters. Because of this we also note that oscillations in the relative difference appear which originate from the baryon acoustic oscillations (BAO) signature imprinted on the matter power spectrum.

We recall that we made the comparison between GGC and $\Lambda \mathrm{CDM}$ respectively with their best-fit parameters with the purpose of spotting differences which can be closer to what we can actually observe. On the contrary in the bottom panel of Fig. 8 . we compare the GGC model with the $\Lambda$ CDM one having the same cosmological parameters of the GGC. In this case any difference we spot can be traced back to modified gravity only. The GGC model is slightly suppressed with respect to $\Lambda \mathrm{CDM}$ on very large scales $k<10^{-3} \mathrm{~h} / \mathrm{Mpc}$. This is due to modifications in the evolution of the linear perturbations. Then, on intermediate scales $\left(10^{-3} h \mathrm{Mpc}^{-1}<k<k_{\mathrm{s}}\right)$ we see an increase of power of about $7 \%-8 \%$. This is a consequence of a stronger gravity force in the GGC model which is given by Eq. (12). Approaching $k_{s}$ the GGC matter power spectrum declines due to the screening effect. The larger is the screening scale, the longer is the plateau. For $k \approx 2 k_{\mathrm{s}}$ the model is fully screened reaching the $\Lambda \mathrm{CDM}$ limit, as expected. Note that when comparing models with the same cosmological parameters, the wiggles in the ratio disappear, as the position of the $\mathrm{BAO}$ wiggles coincide.

We now investigate the effects of the GGC signatures on the lensing power spectrum. The latter is defined as the integral along the line of sight of the matter power spectrum. As for the matter power spectrum, we need to take into account the effects of modifications to gravity and on smaller scales we have to include those of the screening mechanism. The lensing effect depends on the sum of the two gravitational potentials, $\Phi+\Psi$, and as discussed in Sections 3 and 4 any departure form GR in the lensing equation can be included in the phenomenological function $\Sigma$. For GGC $\Phi=\Psi$ even on non-linear scale, so that $\Sigma=\mu$. In the following analysis we assume for $\Sigma$ the functional form [93-95]

$$
\Sigma(k, z)=1+\left(\Sigma^{\mathrm{L}}(z)-1\right) \exp \left[-\left(\frac{k}{k_{\mathrm{s}}}\right)^{2}\right]
$$

The expression used to evaluate the lensing power spectrum is 100

$$
P_{\kappa}(\ell)=\frac{9 H_{0}^{4} \Omega_{\mathrm{m}}^{(0)}}{4 c^{4}} \int_{0}^{\chi_{\mathrm{H}}} \frac{W(\chi)^{2} \Sigma(\chi, k)^{2}}{a^{2}(\chi)} P^{N L}\left[\frac{\ell+1 / 2}{\chi}, \chi\right] \mathrm{d} \chi,
$$

\footnotetext{
${ }^{8}$ With respect to Ref. [100], in Eq. 43] we include the modification to gravity with the function $\Sigma$.
}
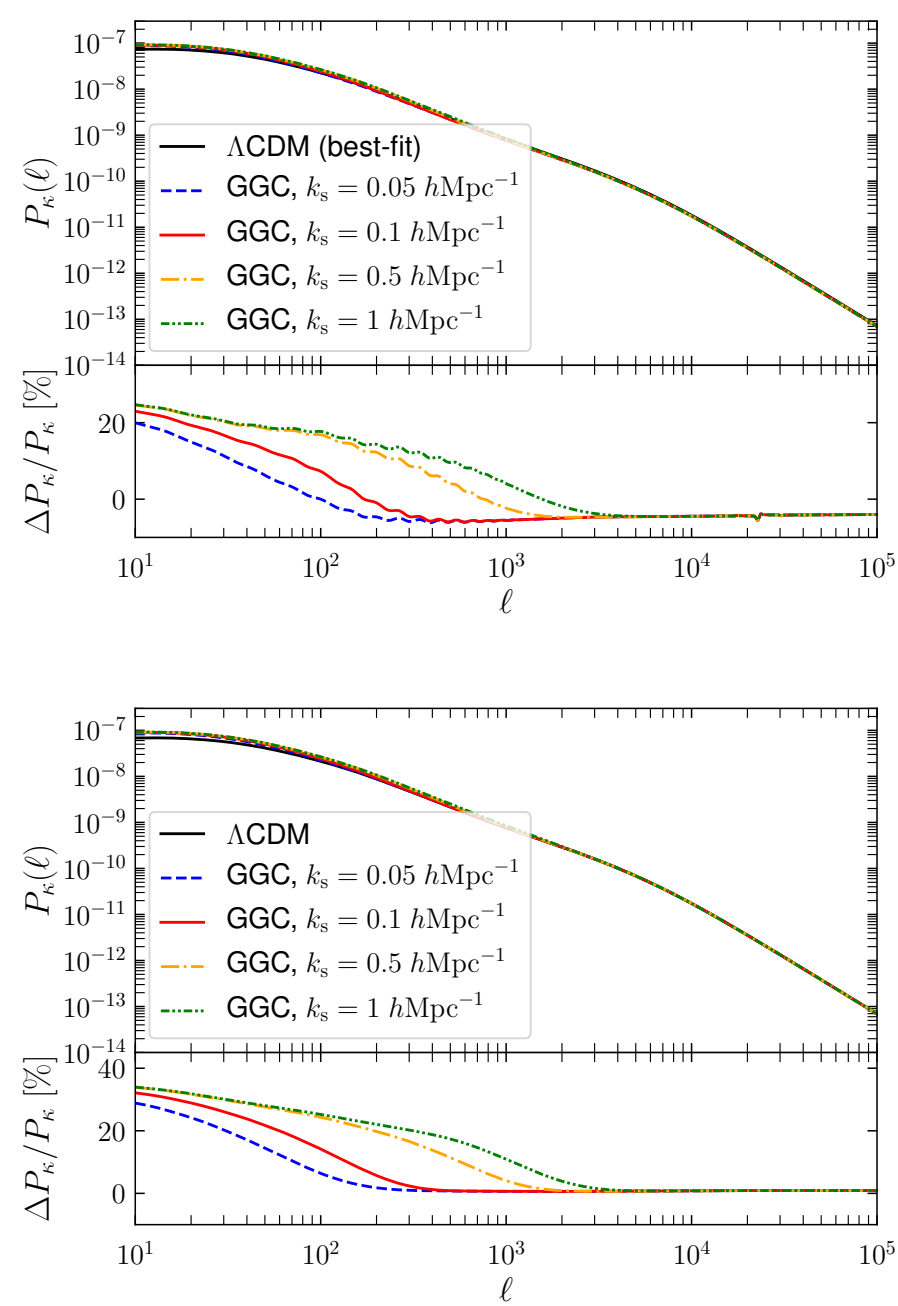

Figure 9: Non-linear lensing power spectra as a function of the multipole $\ell$ for sources at $z_{\mathrm{S}}=2$ for $\Lambda \mathrm{CDM}$ (solid black line) and for the GGC model; and non-linear lensing power spectra percentage relative difference of the GGC model with respect to $\Lambda \mathrm{CDM}$. For the GGC, we show the results for different screening scales, $k_{\mathrm{s}}=0.05,0.1,0.5,1 \mathrm{~h} \mathrm{Mpc}^{-1}$ using the same colour- and linestyle of Fig. 8 The top figure considers the $\Lambda$ CDM and the GGC models with the respective best-fit parameters (Tab. 1, while the bottom one assumes that the $\Lambda$ CDM model has the same cosmological parameters of the GGC model.

where $W(\chi)$ is a kernel describing the distribution in redshift of the sources, $P(k)$ is the matter power spectrum evaluated at the wave-number $k=(\ell+1 / 2) / \chi[101]$, being $\chi$ the comoving distance. Finally, $\chi_{\mathrm{H}}$ represents the comoving distance of the horizon. The function $\Sigma$ depends on the scale $k$ and the time (here parameterized via $\chi$ ). Assuming there is no scale-dependent screening, the expression in Eq. (43) reduces to Eq. (47) of Ref. [102] upon the following identification $\Sigma=1 / F(a)$. Also note that, for simplicity, we assumed the sources to be fixed in redshift at $z_{\mathrm{s}}=2$. Distributing the sources in redshift will not change our conclusions qualitatively, but only slightly decrease the impact of the modifications.

We show in Fig. 9 the results for the non-linear lensing power spectrum in both the $\Lambda \mathrm{CDM}$ and the GGC scenarios. The latter is given for different screening scales. As for the mat- 
ter power spectrum, we compare the two models considering both their best-fit parameters (upper figure) or when they have the same cosmological parameters (lower figure). Because the screening affects small scales (high- $\ell$ ), both models look almost the same in this regime and the multipole where this happen depends, obviously, on the screening scale $k_{\mathrm{s}}$. Larger differences are restricted to small- $\ell$.

Let us start discussing the case in which the two models are characterized by their best-fit parameters (upper panel in Fig. 9]. The GGC lensing power spectrum is enhanced at $\ell=10$ with respect to $\Lambda \mathrm{CDM}$ up to $25 \%$ depending on the particular screening scale. This originates from the parameter $\Sigma(k, z)$ in Eq. (43). Due to the screening, the power decreases linearly until it reaches a plateau for large $\ell$. The rate at which the GGC approaches the plateau is faster for smaller $k_{\mathrm{s}}$, as the screening takes place at larger scales. For the smaller values of the screening scale, it is reached at $\ell \approx 200$, while for $k_{\mathrm{s}}=1 \mathrm{h \textrm {Mpc } ^ { - 1 }}$ it is at $\ell \gtrsim 3000$. For the lensing power spectrum, a change in $k_{\mathrm{s}}$ of a factor of ten changes the scale at which the spectrum of the GGC approaches the plateau roughly by the same amount (see, for example, the behaviour for $k_{\mathrm{s}}=0.1 \mathrm{~h} \mathrm{Mpc}^{-1}$ and $\left.k_{\mathrm{s}}=1 \mathrm{~h} \mathrm{Mpc}^{-1}\right)$. The $5 \%$ suppression in the plateau is due to the different cosmological parameters mostly related to the $\Omega_{\mathrm{m}}^{(0)}$ pre-factor in Eq. 43 which is higher in the $\Lambda$ CDM bestfit case.

Let us now consider the case in which both $\Lambda \mathrm{CDM}$ and GGC share the same cosmological parameters (bottom panel in Fig. 9). The effects of modified gravity are more pronounced at small $\ell$ and this is due to an higher difference in $\Sigma$ with respect to the previous case and it can be up to $35 \%$, a 10\% larger than the best-fit case. At intermediate scale the ripples disappear as expected due to the fact that the matter power spectrum does not show them any more. Finally, when the power spectrum reaches the plateau the discrepancy observed in the previous case disappears as both the power spectrum and $\Sigma$ are in the $\Lambda$ CDM limit and the pre-factor in Eq. 43 (i.e. $\Omega_{\mathrm{m}}^{(0)}$ ) is the same.

\section{Mass function}

In this section we investigate the effects of the GGC model on the abundance of halos. To this purpose we use the Sheth \& Tormen mass function [103-106]

$$
\begin{aligned}
\frac{\mathrm{d} n}{\mathrm{~d} M}= & -\sqrt{\frac{2 \tilde{a}}{\pi}} A\left[1+\left(\frac{\tilde{a} \delta_{\mathrm{c}}^{2}}{D^{2} \sigma_{M}^{2}}\right)^{-p}\right] \frac{\rho_{\mathrm{m}}}{M^{2}} \frac{\delta_{\mathrm{c}}}{D \sigma_{M}} \\
& \times \frac{\mathrm{d} \ln \sigma_{M}}{\mathrm{~d} \ln M} \exp \left(-\frac{\tilde{a} \delta_{\mathrm{c}}^{2}}{2 D^{2} \sigma_{M}^{2}}\right),
\end{aligned}
$$

where $\tilde{a}=0.7079, p=0.3, A=0.2162, \delta_{\mathrm{c}}$ is the linear critical density contrast derived in Section $5, D=\delta_{\mathrm{m}}^{\mathrm{L}} / \delta_{\mathrm{m}}^{\mathrm{L}}(a=1)$ is the linear growth factor, and $\sigma_{M}$ is the variance of the linear matter

\footnotetext{
${ }^{9}$ We changed the commonly adopted notation to avoid confusion with the scale factor $a$.
}
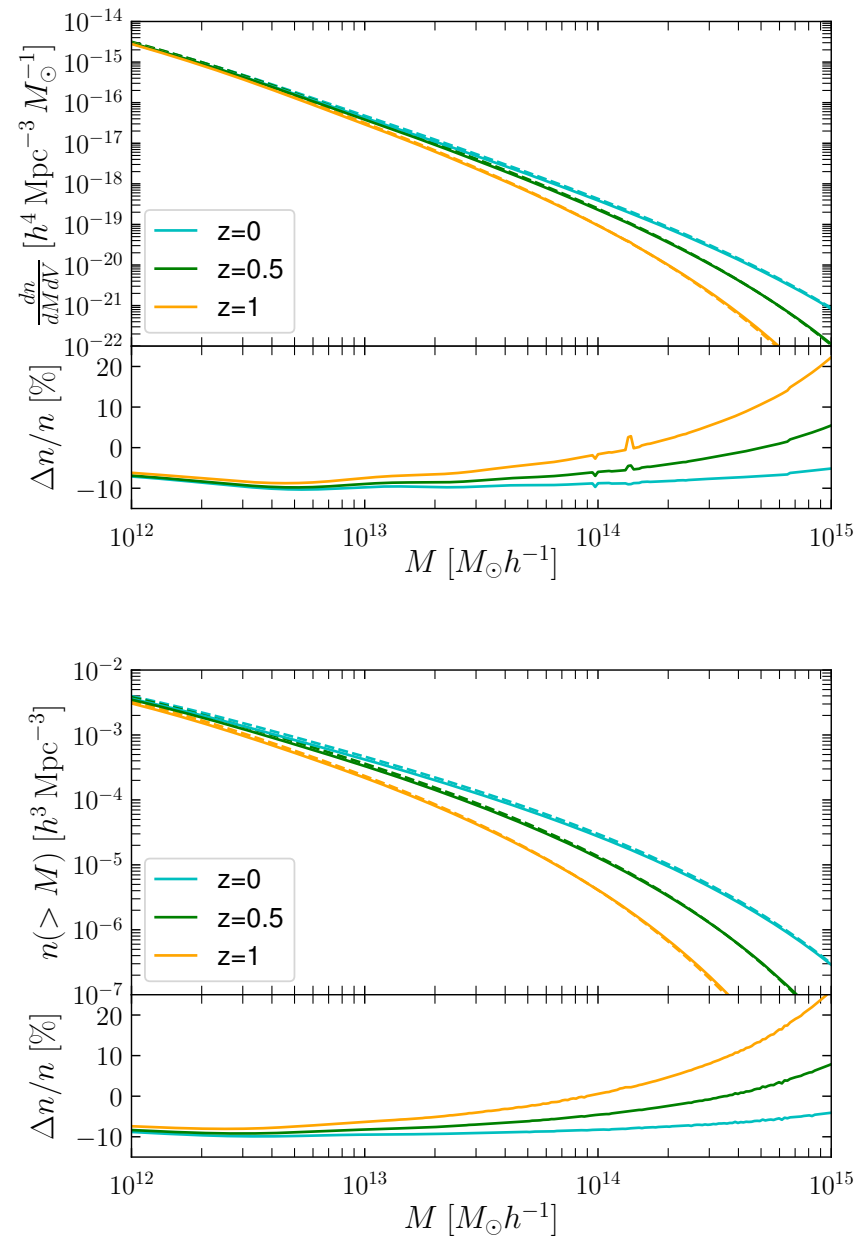

Figure 10: Top panel: differential mass function as a function of the halo mass $M$ at $z=0,0.5,1$, as shown in the labels. Solid lines refer to the GGC model, while dashed lines to $\Lambda \mathrm{CDM}$. Bottom panel: cumulative mass function as a function of the halo mass for the same set of redshifts. The cosmological and models parameters of $\Lambda \mathrm{CDM}$ and GGC models are the best-fit ones in Tab. 1

power spectrum defined as 107

$$
\sigma_{M}^{2}=\frac{1}{2 \pi^{2}} \int_{0}^{\infty} \mathrm{d} k k^{2} W^{2}(k R) P^{L}(k),
$$

where the window function is defined as

$$
W(k R)=3 \frac{\sin (k R)-k R \cos (k R)}{(k R)^{3}},
$$

being $R$ the comoving radius enclosing the mass $M=\frac{4 \pi}{3} \rho_{\mathrm{m}} R^{3}$. The window function represents the Fourier transform of the top-hat function in the space configuration. We also compute the number density of objects above a given mass at a chosen $z$ as:

$$
n(>M)=\int_{M}^{\infty} \frac{\mathrm{d} n}{\mathrm{~d} M^{\prime}} \mathrm{d} M^{\prime} .
$$

In the expression for the mass function, whilst we keep the same constants $(A, \tilde{a}$ and $p)$ for both the GGC and $\Lambda$ CDM, the 

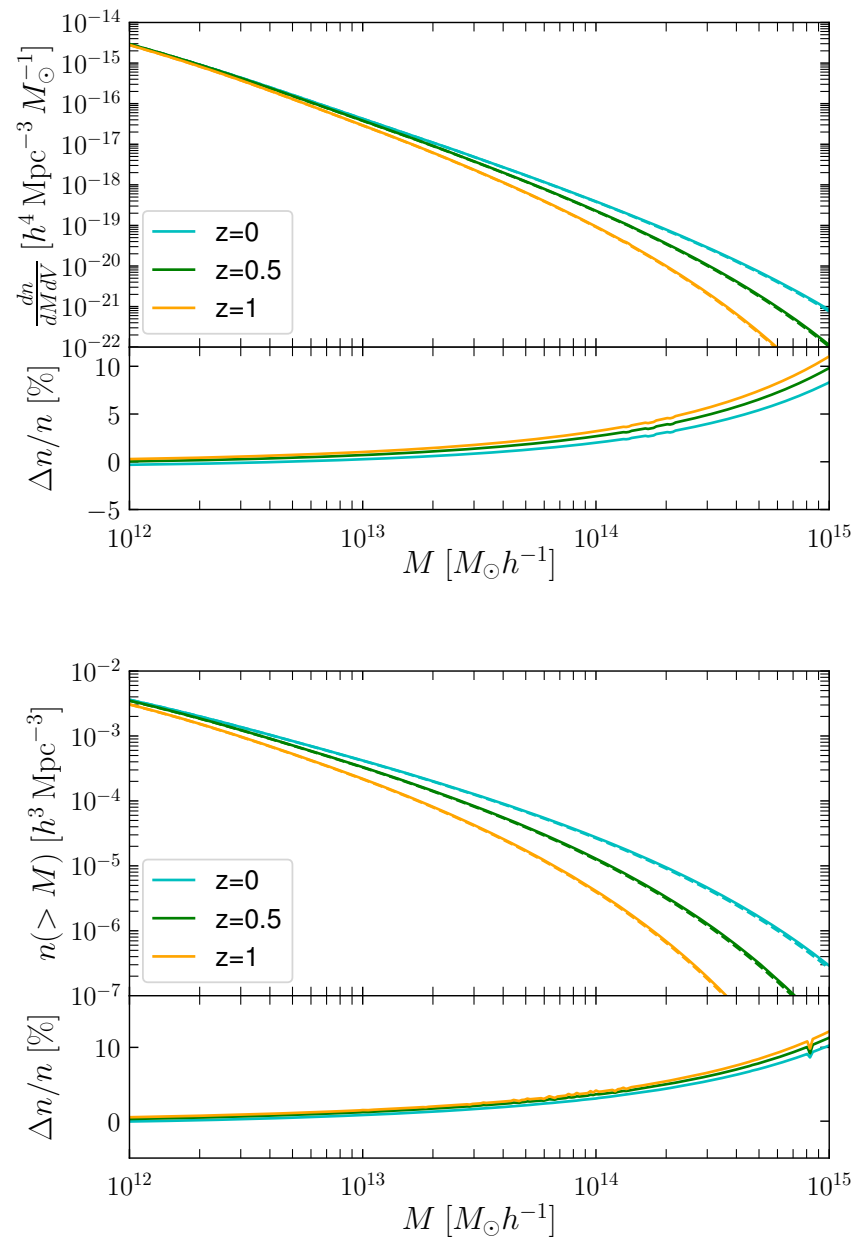

Figure 11: Same as in Fig. 10 but now the $\Lambda \mathrm{CDM}$ shares the same cosmological parameters of the GGC model.

physical parameters $\left(\delta_{\mathrm{c}}, \sigma_{M}, D\right)$ are consistently computed for each model.

We present the results in Fig. 10 and in Fig. 11, where we show the differential and the cumulative mass functions. We also consider the relative difference of the GGC model with respect to $\Lambda \mathrm{CDM}$, i.e. $\Delta n / n=\left(n^{\mathrm{GGC}}-n^{\Lambda \mathrm{CDM}}\right) / n^{\Lambda \mathrm{CDM}}$. We select three redshifts $z=0,0.5,1$ and we consider halo masses ranging from $10^{12} M_{\odot} h^{-1}$ (galactic scales), until $10^{15} M_{\odot} h^{-1}$ (cluster scales) to better assess the effects of GGC at different mass scales and redshifts. In Figs. 10 and 11 we compare GGC and $\Lambda \mathrm{CDM}$ having their best-fit parameters and the same cosmological parameters, respectively.

When comparing the models using their best-fit parameters, for low masses we observe a decrease of about $10 \%$ in the GGC model with respect to $\Lambda \mathrm{CDM}$, regardless of the chosen redshift. At $z=0$, the lack of objects is rather constant over two orders of magnitudes in mass in the two models. The number of objects become more similar towards high masses, but the GGC model still shows a few percent less objects than $\Lambda$ CDM. At higher redshifts, the differences observed at low masses get smaller going towards $M \approx 10^{14} M_{\odot} h^{-1}$ for $z=0.5$ and $M \approx 3 \times$
$10^{13} M_{\odot} h^{-1}$ for $z=1$, respectively, but for higher masses they become more prominent and it is where the two models differ the most in the predictions of the number of halos.

What noticed is the typical behaviour of models beyond $\Lambda \mathrm{CDM}$. The reason is that the exponential suppression in the halo mass function (and, as a consequence, also in the cumulative mass function) is more important at high masses and redshifts [103-105]. The GGC model predicts an excess of objects in the high-mass tail: for $z=0.5$ and $z=1$ we find, respectively, up to $10 \%$ and $20 \%$ more objects. The differences between GGC and $\Lambda \mathrm{CDM}$ models using the best-fit parameters are caused by the substantially different evolution of the linear critical overdensity $\delta_{\mathrm{c}}$ (see Fig. 6) and of the mass variance $\sigma_{M}$ as the linear matter power spectra in the two cosmological models differ by almost $20 \%$ on large scales $\left(k \approx 10^{-4} h \mathrm{Mpc}^{-1}\right)$ and $5 \%-7 \%$ on small scales $\left(k \approx 2 h \mathrm{Mpc}^{-1}\right)$. More in detail, the matter power spectrum in the GGC model is higher than the $\Lambda \mathrm{CDM}$ one, and the same happens for the variance $\sigma_{M}$. This implies that while a higher value of $\delta_{\mathrm{c}}$ leads to a suppression in the mass function, a higher value of $\sigma_{M}$ instead leads to an enhancement. At low redshifts, as the differences due to $\delta_{\mathrm{c}}$ are bigger than those of $\sigma_{M}$ and $\delta_{\mathrm{c}}(\mathrm{GGC})>\delta_{\mathrm{c}}(\Lambda \mathrm{CDM})$, we have a suppression in the number of objects; at high redshifts $\delta_{\mathrm{c}}(\mathrm{GGC}) \gtrsim \delta_{\mathrm{c}}(\Lambda \mathrm{CDM})$ and the major effect is due to the mass variance $\sigma_{M}$, hence we observe an increase of the relative mass function at high masses. At low masses, the exponential term contributes less to the overall picture with respect to the other terms and the decrease in the number of objects is due to the latter.

Instead, when we compare the two models using the same cosmological parameters, they have the same behaviour on small masses and the GGC, due to a higher clustering, predicts more massive halos than the $\Lambda \mathrm{CDM}$. With respect to the best-fit case, differences are slightly smaller (up to $10 \%$ ) and we do not find a significant dependence with redshift. This is in agreement with our previous findings about the matter and lensing power spectra, as small masses pick up the linear part of the matter power spectrum.

From the observational side it would be of interest to compare the predicted halo mass function for the GGC model with the data obtained in Ref. [108] from a sub-sample of 843 clusters (SelFMC) in the redshift range $0.01 \leq z \leq 0.125$ with virial masses of $M \geq 0.8 \times 10^{14} h^{-1} M_{\odot}$ from the GalWCat19 catalogue [109]. As the catalogue is complete in the mass range of $10^{14}<M / M_{\odot}<10^{15}$, where the GGC predictions largely differ from $\Lambda C D M$, it is possible to better assess the influence of modifications of gravity. An extension of the analysis to smaller masses or different redshift ranges, would require to weigh the observed mass function with a selection function $S(D)$, where $D$ is the comoving distance of the cluster. For more details about the procedure, we refer the reader to [108]. Let us open a parenthesis about the impact of any assumption on the underlying cosmological model made to derive the data. For example, since the catalogue includes only objects at small redshift, any influence due a different background evolution can be safely neglected when considering the distance of the clusters and the cosmic volume spanned by the survey as they can be 
approximated with $c / H_{0}$ and $\left(c / H_{0}\right)^{3}$, respectively, where $c$ is the speed of light. However, in the process of mass calibration modifications of gravity might play a role. To this purpose, we can consider, for example, the mass-temperature relation used in [110 112] which reads

$$
\tilde{M}=1.5 \times 10^{14} h^{-1} M_{\odot} \kappa_{\Delta} \frac{T_{X}}{\mathrm{keV}} \frac{1}{1+z},
$$

where $\tilde{M}$ is the virial mass contained in a comoving radius $R_{0}^{\prime}=1.5 h^{-1} \mathrm{Mpc}$ and $T_{X}$ the cluster X-ray temperature. The quantity $\kappa_{\Delta}$ depends on the virial overdensity $\Delta_{\text {vir }}$ which can change in modified gravity cosmologies. In Fig. 7 we showed that $\Delta_{\text {vir }}^{\mathrm{GGC}} \simeq \Delta_{\text {vir }}^{\Lambda \mathrm{CDM}}$ for the best-fit parameter values over all the cosmic history relevant to this work. Hence, the dependence on the modified cosmological model is removed in the case under analysis. On the contrary this will not be the case for G3.

\section{Conclusions}

In this work we studied the impact of non-linearities in the Galileon ghost condensate (GGC) model [31] on the formation of spherical gravitationally bounded objects and we made theoretical predictions on the abundances of halos, non-linear matter and lensing power spectra. To spot key features we have compared the results with the standard cosmological scenario $\Lambda \mathrm{CDM}$ and another Galileon model, the cubic Galileon (G3) [27] which shares with the GGC the term in the Lagrangian $\propto X \square \phi+X$ but differs for the $X^{2}$ term which is not present in the G3. The results presented in the analysis used the maximum likelihood values for the cosmological and model parameters obtained with Planck data in previous works. This is because we wanted to show the difference between the predictions of every model as close as possible to what we actually expect from observations.

We found that the predictions on the growth of structures and spherical collapse of the GGC model are quite different from those of the G3 and are closer to the $\Lambda$ CDM ones but still with some peculiarities. To start with, the linear growth rate in G3 presents large enhancements with respect to the GGC, being the latter very close to $\Lambda \mathrm{CDM}$. On non-linear scales, the presence of the Vainshtein screening mechanism which characterises both Galileon models changes the gravitational coupling felt by matter which, in both cases, is larger than that in $\Lambda \mathrm{CDM}$. We noted that for a collapse taking place at the present time in the GGC model such gravitational coupling stays closer to $\Lambda \mathrm{CDM}$ than the G3 one. This is due to the fact that the former enters in the Vainshtein radius before. Indeed, during the collapse process, the Vainshtein radius of the GGC is always larger than that in the G3. Furthermore, we found that the turnaround phase for both Galileon models takes place slightly before than for $\Lambda \mathrm{CDM}$ and the virialization time follows the order $\mathrm{G} 3, \mathrm{GGC}$ and $\Lambda \mathrm{CDM}$. Being $\mathrm{G} 3$ the first to reach virialization, the evolution of the virial overdensity for the Galileon models is completely different: while G3 stays always below $\Lambda \mathrm{CDM}$, the GGC closely follows the $\Lambda \mathrm{CDM}$ one and after $a \approx 0.5$ it is slightly enhanced. The evolution of the linear critical overdensity $\delta_{\mathrm{c}}$ shows again key features after $z \approx 2$ : in the $\Lambda$ CDM scenario it decreases from the de-Sitter value to $\approx 1.675$ at present time; in the GGC, instead, it has the opposite behaviour, increasing its value up to 1.708; finally the G3 grows as well but up to $a \approx 0.7$ it stays below the GGC and after it overcomes the GGC, reaching the present day value of $\approx 1.74$. These new features of the GGC can be addressed considering the inclusion of the $X^{2}$ term in the Lagrangian which makes the difference with respect to G3. This term changes remarkably the evolution of the Vainshtein radius and as such the physics associated to the formation of (non-linear) structures.

We employed a phenomenological approach to incorporate the screening mechanism in the computation of the non-linear matter and lensing power spectra. This is done by considering the gravitational couplings felt by matter and light, respectively, to have an explicit dependence on the screening scale, $k_{\mathrm{s}}$ such that when $k<k_{\mathrm{s}}$ it reduces to the linear GGC spectrum while when $k>k_{\mathrm{s}}$ they approach the $\Lambda \mathrm{CDM}$ behaviour. Because we do not know the screening scale of the GGC model in Fourier space, we computed the predictions for matter and lensing power spectra for four scales. This approach led us to show the phenomenology of GGC and provide theoretical predictions which, in the future, can be compared to accurate $N$-body simulations once they are available. We found that when comparing the models characterized by their best-fit parameters, the matter power spectrum on linear scales for GGC shows a difference with respect to $\Lambda \mathrm{CDM}$ smaller than $20 \%$ and it decreases to a few percent on the smaller scales. The scale at which the matter power spectrum approaches a plateau on large $k$ depends on the screening scale. As expected, smaller screening scales suppress the matter power spectrum at larger scales. When we compare the models using the same base cosmological parameters the difference in the matter power spectrum persists only on intermediate scales $10^{-3} h \mathrm{Mpc}^{-1}<k<k_{\mathrm{s}}$ (up to $7 \%-8 \%$ ). In the lensing power spectrum, the relative difference between GGC and $\Lambda$ CDM for the best-fit case, exceeds $20 \%$ at small- $\ell$ and decreases at larger- $\ell$. The values of $k_{\mathrm{s}}$ we chose show that for the smaller value of $k_{\mathrm{s}}=0.05 \mathrm{~h} \mathrm{Mpc}^{-1}$, the $\Lambda \mathrm{CDM}$ limit is reached at $\ell \approx 200$ and for the larger $k_{\mathrm{s}}=1 \mathrm{~h} \mathrm{Mpc}^{-1}$ we found $\ell \approx 3000$. A similar behaviour characterizes the comparison when the same cosmological parameters are employed, but in this case the difference at small $\ell$ is $10 \%$ larger. We then computed the mass function as a function of the halo mass following the Sheth \& Tormen model. We found that for the best-fit case at low masses the GGC model provides about $10 \%$ less objects with respect to $\Lambda \mathrm{CDM}$, while at higher masses and higher redshift $(z>0.5)$ it predicts about $10 \%-20 \%$ more objects. This can be explained by the fact that the critical linear overdensity for GGC is larger than in $\triangle \mathrm{CDM}$ and by a larger mass variance in the former. When the difference due to the different cosmological parameters is removed we found that at small masses both models predict the same number of objects but at larger masses GGC predicts up to $10 \%$ more objects than $\Lambda \mathrm{CDM}$ regardless of the redshift.

Finally, given the results presented in this paper, the GGC model shows very peculiar and measurable features which can 
definitely help in discriminating between GGC and $\Lambda \mathrm{CDM}$. Whilst this work provides only a glimpse into the phenomenology of non-linear matter and lensing power spectra, less simplified methods can be employed, such as those in Refs. [88, 90, 92], which we will consider in an upcoming work.

We further stress that a proper assessment and validation of our results can come with realistic $N$-body simulations, which are not affected by the necessary simplifications required for an analytical evaluation. Simulations will also allow to produce fitting formulae for the evolution of the non-linear matter power spectrum and improve the formalism of the spherical collapse model.

\section{Acknowledgements}

We thank Alberto Rozas-Fernández, Björn Malte Schäfer and Shinji Tsujikawa for useful discussions. The research of NF is supported by Fundação para a Ciência e a Tecnologia (FCT) through the research grants UID/FIS/04434/2019, UIDB/04434 /2020 and UIDP/04434/2020 and by FCT project "DarkRipple - Spacetime ripples in the dark gravitational Universe" with ref. number PTDC/FIS-OUT/29048/2017. FP acknowledges support from Science and Technology Facilities Council (STFC) grant ST/P000649/1 and the ERC Consolidator Grant $C M B$ SPEC (No. 725456) as part of the European Union's Horizon 2020 research and innovation program.

\section{References}

[1] A. G. Riess, et al. (Supernova Search Team), Observational evidence from supernovae for an accelerating universe and a cosmological constant, Astron. J. 116 (1998) 1009-1038. doi $10.1086 / 300499$ arXiv:astro-ph/9805201

[2] S. Perlmutter, et al. (Supernova Cosmology Project), Measurements of $\Omega$ and $\Lambda$ from 42 high redshift supernovae, Astrophys. J. 517 (1999) 565-586. doi 10.1086/307221 arXiv: astro-ph/9812133

[3] M. Betoule, et al. (SDSS), Improved cosmological constraints from a joint analysis of the SDSS-II and SNLS supernova samples, Astron. Astrophys. 568 (2014) A22. doi $10.1051 / 0004-6361 / 201423413$ arXiv:1401.4064

[4] D. N. Spergel, et al. (WMAP), First year Wilkinson Microwave Anisotropy Probe (WMAP) observations: Determination of cosmological parameters, Astrophys. J. Suppl. 148 (2003) 175-194. doi 10 . $1086 / 377226$ arXiv:astro-ph/0302209

[5] P. A. R. Ade, et al. (Planck), Planck 2015 results. XIII. Cosmological parameters, Astron. Astrophys. 594 (2016) A13. doi $10.1051 /$ 0004-6361/201525830 arXiv:1502.01589

[6] N. Aghanim, et al. (Planck), Planck 2015 results. XI. CMB power spectra, likelihoods, and robustness of parameters, Astron. Astrophys. 594 (2016) A11. doi $10.1051 / 0004-6361 / 201526926$ arXiv:1507.02704

[7] D. J. Eisenstein, et al. (SDSS), Detection of the Baryon Acoustic Peak in the Large-Scale Correlation Function of SDSS Luminous Red Galaxies, Astrophys. J. 633 (2005) 560-574. doi $10.1086 / 466512$ arXiv:astro-ph/0501171

[8] F. Beutler, C. Blake, M. Colless, D. H. Jones, L. Staveley-Smith, L. Campbell, Q. Parker, W. Saunders, F. Watson, The 6dF Galaxy Survey: Baryon Acoustic Oscillations and the Local Hubble Constant, Mon. Not. Roy. Astron. Soc. 416 (2011) 3017-3032. doi $10.1111 / j$. 1365-2966.2011.19250.x arXiv:1106.3366

[9] A. Joyce, B. Jain, J. Khoury, M. Trodden, Beyond the Cosmological Standard Model, Phys. Rept. 568 (2015) 1-98. doi $10.1016 / j$. physrep.2014.12.002 arXiv:1407.0059
[10] A. Lue, R. Scoccimarro, G. D. Starkman, Probing Newton's constant on vast scales: DGP gravity, cosmic acceleration and large scale structure, Phys. Rev. D69 (2004) 124015. doi $10.1103 /$ PhysRevD . 69. 124015 arXiv:astro-ph/0401515

[11] E. J. Copeland, M. Sami, S. Tsujikawa, Dynamics of dark energy, Int. J. Mod. Phys. D15 (2006) 1753-1936. doi $10.1142 /$ S021827180600942X arXiv: hep-th/0603057

[12] A. Silvestri, M. Trodden, Approaches to Understanding Cosmic Acceleration, Rept. Prog. Phys. 72 (2009) 096901. doi $10.1088 /$ 0034-4885/72/9/096901 arXiv:0904.0024

[13] S. Capozziello, M. De Laurentis, Extended Theories of Gravity, Phys. Rept. 509 (2011) 167-321. doi $10.1016 / j$.physrep.2011.09. 003 arXiv:1108.6266

[14] T. Clifton, P. G. Ferreira, A. Padilla, C. Skordis, Modified Gravity and Cosmology, Phys. Rept. 513 (2012) 1-189. doi 10.1016/j. physrep.2012.01.001 arXiv:1106.2476

[15] S. Tsujikawa, Modified gravity models of dark energy, Lect. Notes Phys. 800 (2010) 99-145. doi $10.1007 / 978-3-642-10598-2 \backslash$ 3 arXiv:1101.0191

[16] K. Koyama, Cosmological Tests of Modified Gravity, Rept. Prog. Phys. 79 (2016) 046902. doi $10.1088 / 0034-4885 / 79 / 4 /$ 046902 arXiv:1504.04623

[17] S. Nojiri, S. D. Odintsov, V. K. Oikonomou, Modified gravity theories on a nutshell: Inflation, bounce and late-time evolution, Physics Reports 692 (2017) 1-104. doi $10.1016 / j \cdot p h y s r e p .2017 .06$. 001 arXiv:1705.11098

[18] P. G. Ferreira, Cosmological Tests of Gravity, Ann. Rev. Astron. Astrophys. 57 (2019) 335-374. doi 10.1146/ annurev-astro-091918-104423 arXiv:1902.10503

[19] T. Kobayashi, Horndeski theory and beyond: a review, Reports on Progress in Physics 82 (2019) 086901. doi $10.1088 / 1361-6633 /$ ab2429 arXiv:1901.07183

[20] M. Ishak, Testing general relativity in cosmology, Living Reviews in Relativity 22 (2019) 1. doi 10.1007/s41114-018-0017-4 arXiv:1806.10122

[21] G. W. Horndeski, Second-order scalar-tensor field equations in a fourdimensional space, Int. J. Theor. Phys. 10 (1974) 363-384. doi 10 . $1007 / \mathrm{BF} 01807638$

[22] Y. Fujii, K. Maeda, The scalar-tensor theory of gravitation, Cambridge Monographs on Mathematical Physics, Cambridge University Press, 2007. URL: http://www.cambridge.org/ uk/catalogue/catalogue.asp?isbn=0521811597 doi $10.1017 /$ CBO9780511535093

[23] C. Deffayet, S. Deser, G. Esposito-Farese, Generalized Galileons: All scalar models whose curved background extensions maintain secondorder field equations and stress-tensors, Phys. Rev. D80 (2009) 064015. doi $10.1103 /$ PhysRevD .80.064015 arXiv:0906.1967

[24] J. Gleyzes, D. Langlois, F. Piazza, F. Vernizzi, Healthy theories beyond Horndeski, Phys. Rev. Lett. 114 (2015) 211101. doi $10.1103 /$ PhysRevLett.114.211101 arXiv:1404.6495

[25] D. Langlois, K. Noui, Degenerate higher derivative theories beyond Horndeski: evading the Ostrogradski instability, JCAP 1602 (2016) 034. doi $10.1088 / 1475-7516 / 2016 / 02 / 034$ arXiv:1510.06930

[26] T. Kobayashi, M. Yamaguchi, J. Yokoyama, Generalized G-inflation: Inflation with the most general second-order field equations, Prog. Theor. Phys. 126 (2011) 511-529. doi $10.1143 /$ PTP.126.511 arXiv: 1105.5723

[27] C. Deffayet, G. Esposito-Farese, A. Vikman, Covariant Galileon, Phys. Rev. D79 (2009) 084003. doi $10.1103 /$ PhysRevD.79.084003 arXiv:0901.1314

[28] C. Deffayet, O. Pujolas, I. Sawicki, A. Vikman, Imperfect Dark Energy from Kinetic Gravity Braiding, JCAP 10 (2010) 026. doi $10.1088 /$ 1475-7516/2010/10/026 arXiv:1008.0048

[29] A. De Felice, S. Tsujikawa, Conditions for the cosmological viability of the most general scalar-tensor theories and their applications to extended Galileon dark energy models, JCAP 1202 (2012) 007. doi $10.1088 /$ 1475-7516/2012/02/007 arXiv:1110.3878

[30] A. R. Gomes, L. Amendola, Towards scaling cosmological solutions with full coupled Horndeski Lagrangian: the KGB model, JCAP 1403 (2014) 041. doi:10.1088/1475-7516/2014/03/ 
041 arXiv:1306.3593

[31] R. Kase, S. Tsujikawa, Dark energy scenario consistent with GW170817 in theories beyond Horndeski gravity, Phys. Rev. D97 (2018) 103501. doi 10.1103/PhysRevD.97.103501 arXiv:1802.02728

[32] I. S. Albuquerque, N. Frusciante, N. J. Nunes, S. Tsujikawa, New scaling solutions in cubic Horndeski theories, Phys. Rev. D98 (2018) 064038. doi $10.1103 /$ PhysRevD.98.064038 arXiv: 1807.09800

[33] N. Frusciante, R. Kase, N. J. Nunes, S. Tsujikawa, Most general cubicorder Horndeski Lagrangian allowing for scaling solutions and the application to dark energy, Phys. Rev. D98 (2018) 123517. doi 10.1103/ PhysRevD.98.123517 arXiv:1810.07957

[34] R. Kase, S. Tsujikawa, Dark energy in Horndeski theories after GW170817: A review, Int. J. Mod. Phys. D28 (2019) 1942005. doi $10.1142 /$ S0218271819420057 arXiv:1809.08735

[35] A. De Felice, S. Tsujikawa, Cosmological constraints on extended Galileon models, JCAP 1203 (2012) 025. doi $10.1088 /$ 1475-7516/2012/03/025 arXiv:1112.1774

[36] A. Barreira, B. Li, A. Sanchez, C. M. Baugh, S. Pascoli, Parameter space in Galileon gravity models, Phys. Rev. D87 (2013) 103511. doi 10 . 1103/PhysRevD . 87.103511 arXiv:1302.6241

[37] A. Barreira, B. Li, C. Baugh, S. Pascoli, The observational status of Galileon gravity after Planck, JCAP 1408 (2014) 059. doi $10.1088 /$ 1475-7516/2014/08/059 arXiv:1406.0485

[38] J. Renk, M. Zumalacarregui, F. Montanari, A. Barreira, Galileon gravity in light of ISW, $\mathrm{CMB}, \mathrm{BAO}$ and $\mathrm{H}_{0}$ data, JCAP 1710 (2017) 020. doi $10.1088 / 1475-7516 / 2017 / 10 / 020$ arXiv:1707.02263

[39] S. Peirone, N. Frusciante, B. Hu, M. Raveri, A. Silvestri, Do current cosmological observations rule out all Covariant Galileons?, Phys. Rev. D97 (2018) 063518. doi $10.1103 /$ PhysRevD.97.063518 arXiv:1711.04760

[40] S. Peirone, G. Benevento, N. Frusciante, S. Tsujikawa, Cosmological data favor Galileon ghost condensate over $\Lambda \mathrm{CDM}$, Phys. Rev. D100 (2019) 063540. doi 10.1103 /PhysRevD. 100.063540 arXiv:1905.05166

[41] F. Giacomello, A. De Felice, S. Ansoldi, Bounds from ISWgalaxy cross-correlations on generalized covariant Galileon models, JCAP 1903 (2019) 038. doi/10.1088/1475-7516/2019/03/ 038 arXiv: 1811.10885

[42] N. Frusciante, S. Peirone, L. Atayde, A. De Felice, Phenomenology of the generalized cubic covariant Galileon model and cosmological bounds, Phys. Rev. D101 (2020) 064001. doi 10.1103/PhysRevD . 101.064001 arXiv: 1912.07586

[43] A. I. Vainshtein, To the problem of nonvanishing gravitation mass, Phys. Lett. 39B (1972) 393-394. doi $10.1016 / 0370-2693(72)$ 90147-5

[44] A. Nicolis, R. Rattazzi, E. Trincherini, The Galileon as a local modification of gravity, Phys. Rev. D79 (2009) 064036. doi 10.1103/ PhysRevD.79.064036 arXiv:0811.2197

[45] K. Koyama, G. Niz, G. Tasinato, Effective theory for the Vainshtein mechanism from the Horndeski action, Phys. Rev. D88 (2013) 021502. doi 10.1103/PhysRevD.88.021502 arXiv:1305.0279

[46] R. Kimura, T. Kobayashi, K. Yamamoto, Vainshtein screening in a cosmological background in the most general second-order scalar-tensor theory, Phys. Rev. D85 (2012) 024023. doi 10.1103/PhysRevD. 85.024023 arXiv: 1111.6749

[47] J.-P. Uzan, Varying constants, gravitation and cosmology, Living Reviews in Relativity 14 (2011) 2. URL:/https://doi.org/10. 12942/lrr-2011-2 doi 10.12942/lrr-2011-2

[48] C. M. Will, The confrontation between general relativity and experiment, Living Reviews in Relativity 17 (2014) 4. URL: https : / / doi . org/10.12942/lrr-2014-4 doi 10.12942/lrr-2014-4

[49] F. Schmidt, W. Hu, M. Lima, Spherical Collapse and the Halo Model in Braneworld Gravity, Phys. Rev. D81 (2010) 063005. doi 10.1103/ PhysRevD.81.063005 arXiv:0911.5178

[50] R. Kimura, K. Yamamoto, Large Scale Structures in Kinetic Gravity Braiding Model That Can Be Unbraided, JCAP 1104 (2011) 025 doi 10.1088/1475-7516/2011/04/025 arXiv:1011.2006

[51] E. Bellini, N. Bartolo, S. Matarrese, Spherical Collapse in covariant Galileon theory, JCAP 1206 (2012) 019. doi $10.1088 / 1475-7516 /$
2012/06/019 arXiv:1202.2712

[52] A. Barreira, B. Li, W. A. Hellwing, C. M. Baugh, S. Pascoli, Nonlinear structure formation in the Cubic Galileon gravity model, JCAP 1310 (2013) 027. doi $10.1088 / 1475-7516 / 2013 / 10 /$ 027 arXiv: 1306.3219

[53] LSST Science Collaboration, LSST Science Book, Version 2.0, arXiv e-prints (2009) arXiv:0912.0201.arXiv:0912.0201

[54] LSST Dark Energy Science Collaboration, Large Synoptic Survey Telescope: Dark Energy Science Collaboration, arXiv e-prints (2012) arXiv:1211.0310. arXiv:1211.0310

[55] DESI Collaboration, et al., The DESI Experiment Part I: Science,Targeting, and Survey Design, arXiv e-prints (2016) arXiv:1611.00036. arXiv:1611.00036

[56] R. Laureijs, Euclid Assessment Study Report for the ESA Cosmic Visions, ArXiv e-prints, 0912.0914 (2009). arXiv: 0912.0914

[57] R. Laureijs, et al. (EUCLID), Euclid Definition Study Report (2011). arXiv: 1110.3193

[58] A. Weltman, P. Bull, S. Camera, K. Kelley, H. Padmanabhan, J. Pritchard, A. Raccanelli, S. Riemer-Sørensen, L. Shao, et al., Fundamental physics with the Square Kilometre Array, Publ. Astron. Soc. Austral. 37 (2020) e002. doi 10.1017/pasa.2019.42 arXiv: 1810.02680

[59] L. Amendola, M. Kunz, D. Sapone, Measuring the dark side (with weak lensing), JCAP 0804 (2008) 013. doi 10 .1088/1475-7516/2008/ 04/013 arXiv:0704.2421

[60] E. Bertschinger, P. Zukin, Distinguishing Modified Gravity from Dark Energy, Phys. Rev. D78 (2008) 024015. doi 10.1103/PhysRevD. 78.024015 arXiv:0801.2431

[61] L. Pogosian, A. Silvestri, K. Koyama, G.-B. Zhao, How to optimally parametrize deviations from General Relativity in the evolution of cosmological perturbations?, Phys. Rev. D81 (2010) 104023. doi 10.1103/PhysRevD.81.104023 arXiv:1002.2382

[62] S. Peirone, K. Koyama, L. Pogosian, M. Raveri, A. Silvestri, Largescale structure phenomenology of viable Horndeski theories, Phys. Rev. D97 (2018) 043519. doi $10.1103 /$ PhysRevD.97.043519 arXiv: 1712.00444

[63] N. Frusciante, S. Peirone, S. Casas, N. A. Lima, Cosmology of surviving Horndeski theory: The road ahead, Phys. Rev. D99 (2019) 063538. doi 10.1103/PhysRevD.99.063538 arXiv:1810.10521

[64] B. Boisseau, G. Esposito-Farese, D. Polarski, A. A. Starobinsky, Reconstruction of a scalar tensor theory of gravity in an accelerating universe, Phys. Rev. Lett. 85 (2000) 2236. doi 10.1103/PhysRevLett. 85. 2236 arXiv:gr-qc/0001066

[65] A. De Felice, T. Kobayashi, S. Tsujikawa, Effective gravitational couplings for cosmological perturbations in the most general scalar-tensor theories with second-order field equations, Phys. Lett. B706 (2011) 123-133. doi $10.1016 / j . p h y s l e t b .2011$. 11.028 arXiv: 1108.4242

[66] I. Sawicki, E. Bellini, Limits of quasistatic approximation in modifiedgravity cosmologies, Phys. Rev. D92 (2015) 084061. doi 10.1103/ PhysRevD.92.084061 arXiv:1503.06831

[67] A. De Felice, S. Tsujikawa, Cosmology of a covariant Galileon field, Phys. Rev. Lett. 105 (2010) 111301. doi 10.1103/PhysRevLett. 105.111301 arXiv:1007.2700

[68] O. Lahav, P. B. Lilje, J. R. Primack, M. J. Rees, Dynamical effects of the cosmological constant, Mon. Not. Roy. Astron. Soc. 251 (1991) $128-136$.

[69] F. Pace, C. Schimd, D. F. Mota, A. Del Popolo, Halo collapse: virialization by shear and rotation in dynamical dark-energy models. Effects on weak-lensing peaks, JCAP 2019 (2019) 060. doi $10.1088 /$ 1475-7516/2019/09/060 arXiv:1811.12105

[70] F. Pace, J.-C. Waizmann, M. Bartelmann, Spherical collapse model in dark-energy cosmologies, MNRAS 406 (2010) 1865-1874. doi 10 . 1111/j.1365-2966.2010.16841.x arXiv:1005.0233

[71] F. Pace, S. Meyer, M. Bartelmann, On the implementation of the spherical collapse model for dark energy models, JCAP 10 (2017) 040. doi $10.1088 / 1475-7516 / 2017 / 10 / 040$ arXiv: 1708.02477

[72] E. Bellini, et al., Comparison of Einstein-Boltzmann solvers for testing general relativity, Phys. Rev. D97 (2018) 023520. doi $10.1103 /$ PhysRevD.97.023520 arXiv:1709.09135 
[73] H. Oyaizu, Nonlinear evolution of $f(R)$ cosmologies. I. Methodology, Phys. Rev. D 78 (2008) 123523. doi 10.1103 /PhysRevD . 78 . 123523 arXiv:0807.2449

[74] F. Schmidt, Cosmological simulations of normal-branch braneworld gravity, Phys. Rev. D 80 (2009) 123003. doi 10.1103/PhysRevD . 80.123003 arXiv:0910.0235

[75] G.-B. Zhao, B. Li, K. Koyama, N-body simulations for $\mathrm{f}(\mathrm{R})$ gravity using a self-adaptive particle-mesh code, Phys. Rev. D 83 (2011) 044007. doi 10.1103/PhysRevD.83.044007 arXiv:1011.1257

[76] B. Li, G.-B. Zhao, R. Teyssier, K. Koyama, ECOSMOG: an Efficient COde for Simulating MOdified Gravity, JCAP 2012 (2012) 051. doi 10.1088/1475-7516/2012/01/051 arXiv:1110.1379

[77] P. Brax, A.-C. Davis, B. Li, H. A. Winther, G.-B. Zhao, Systematic simulations of modified gravity: symmetron and dilaton models, JCAP 2012 (2012) 002. doi $10.1088 / 1475-7516 / 2012 / 10 /$ 002 arXiv:1206.3568

[78] P. Brax, A.-C. Davis, B. Li, H. A. Winther, Unified description of screened modified gravity, Phys. Rev. D 86 (2012) 044015. doi 10 . 1103/PhysRevD.86.044015 arXiv:1203.4812

[79] M. Baldi, Dark Energy simulations, Physics of the Dark Universe 1 (2012) 162-193. doi $10.1016 / j$. dark.2012.10.004 arXiv: 1210.6650

[80] E. Puchwein, M. Baldi, V. Springel, Modified-Gravity-GADGET: a new code for cosmological hydrodynamical simulations of modified gravity models, MNRAS 436 (2013) 348-360. doi $10.1093 / \mathrm{mnras} /$ stt1575 arXiv:1305.2418

[81] M. Wyman, E. Jennings, M. Lima, Simulations of Galileon modified gravity: Clustering statistics in real and redshift space, Phys. Rev. D 88 (2013) 084029. doi $10.1103 /$ PhysRevD.88.084029 arXiv: 1303.6630

[82] B. Li, A. Barreira, C. M. Baugh, W. A. Hellwing, K. Koyama, S. Pascoli, G.-B. Zhao, Simulating the quartic Galileon gravity model on adaptively refined meshes, JCAP 2013 (2013) 012. doi 10.1088/1475-7516/ 2013/11/012 arXiv:1308.3491

[83] C. Llinares, D. F. Mota, H. A. Winther, ISIS: a new N-body cosmological code with scalar fields based on RAMSES. Code presentation and application to the shapes of clusters, A\&A 562 (2014) A78. doi $10.1051 / 0004-6361 / 201322412$ arXiv:1307.6748

[84] C. Llinares, D. F. Mota, Cosmological simulations of screened modified gravity out of the static approximation: Effects on matter distribution, Phys. Rev. D 89 (2014) 084023. doi 10.1103/PhysRevD . 89. 084023 arXiv:1312.6016

[85] C. Llinares, Simulation techniques for modified gravity, International Journal of Modern Physics D 27 (2018) 1848003. doi $10.1142 /$ S0218271818480036

[86] B. Li, Simulating Large-Scale Structure for Models of Cosmic Acceleration, 2018. doi $10.1088 / 978-0-7503-1587-6$

[87] C. Llinares, R. Hagala, D. F. Mota, Non-linear phenomenology of disformally coupled quintessence, MNRAS 491 (2020) 1868-1886. doi $10.1093 / \mathrm{mnras} / \mathrm{stz} 2710$ arXiv:1902.02125

[88] K. Koyama, A. Taruya, T. Hiramatsu, Non-linear Evolution of Matter Power Spectrum in Modified Theory of Gravity, Phys. Rev. D79 (2009) 123512. doi 10.1103/PhysRevD.79.123512 arXiv:0902.0618

[89] A. Taruya, T. Hiramatsu, A Closure Theory for Non-linear Evolution of Cosmological Power Spectra, Astrophys. J. 674 (2008) 617. doi 10 . 1086/526515 arXiv:0708.1367

[90] M. Cataneo, L. Lombriser, C. Heymans, A. Mead, A. Barreira, S. Bose, B. $\mathrm{Li}$, On the road to percent accuracy: non-linear reaction of the matter power spectrum to dark energy and modified gravity, Mon. Not. Roy. Astron. Soc. 488 (2019) 2121-2142. doi $10.1093 / \mathrm{mnras} /$ stz1836 arXiv: 1812.05594

[91] A. Mead, Spherical collapse, formation hysteresis and the deeply non-linear cosmological power spectrum, Mon. Not. Roy. Astron. Soc. 464 (2017) 1282-1293. doi $10.1093 / \mathrm{mnras} / \mathrm{stw} 2312$ arXiv: 1606.05345

[92] F. Hassani, L. Lombriser, $N$-body simulations for parametrised modified gravity (2020). arXiv: 2003.05927

[93] D. Alonso, E. Bellini, P. G. Ferreira, M. Zumalacarregui, Observational future of cosmological scalar-tensor theories, Phys Rev. D95 (2017) 063502. doi $10.1103 /$ PhysRevD.95.063502
arXiv: 1610.09290

[94] M. Fasiello, Z. Vlah, Screening in perturbative approaches to LSS, Phys. Lett. B 773 (2017) 236-241. doi $10.1016 / j . p h y s l e t b .2017$. 08.032 arXiv: 1704.07552

[95] R. Reischke, A. Spurio Mancini, B. M. SchÃd'fer, P. M. Merkel, Investigating scalarâASŞtensor gravity with statistics of the cosmic largescale structure, Mon. Not. Roy. Astron. Soc. 482 (2019) 3274-3287. doi $10.1093 / \mathrm{mnras} / \mathrm{sty} 2919$ arXiv:1804.02441

[96] A. Lewis, A. Challinor, A. Lasenby, Efficient computation of CMB anisotropies in closed FRW models, Astrophys. J. 538 (2000) 473-476. doi $10.1086 / 309179$ arXiv: astro-ph/9911177

[97] M. Raveri, B. Hu, N. Frusciante, A. Silvestri, Effective Field Theory of Cosmic Acceleration: constraining dark energy with CMB data, Phys. Rev. D90 (2014) 043513. doi $10.1103 /$ PhysRevD.90.043513 arXiv:1405.1022

[98] B. Hu, M. Raveri, A. Silvestri, N. Frusciante, Exploring massive neutrinos in dark cosmologies with EFTCAMB/ EFTCosmoMC, Phys. Rev. D91 (2015) 063524. doi 10.1103/PhysRevD.91.063524 arXiv:1410.5807

[99] R. E. Smith, J. A. Peacock, A. Jenkins, S. D. M. White, C. S. Frenk, F. R. Pearce, P. A. Thomas, G. Efstathiou, H. M. P. Couchman, Stable clustering, the halo model and non-linear cosmological power spectra, MNRAS 341 (2003) 1311-1332. doi $10.1046 / j .1365-8711$. $2003.06503 . x$ arXiv:astro-ph/0207664

[100] M. Bartelmann, P. Schneider, Weak gravitational lensing, Physics Reports 340 (2001) 291-472. doi 10.1016/S0370-1573(00) 00082-X arXiv:arXiv:astro-ph/9912508

[101] M. Loverde, N. Afshordi, Extended Limber approximation, Phys. Rev. D 78 (2008) 123506. doi $10.1103 /$ PhysRevD.78.123506 arXiv:0809.5112

[102] F. Pace, L. Moscardini, R. Crittenden, M. Bartelmann, V. Pettorino, A comparison of structure formation in minimally and non-minimally coupled quintessence models, MNRAS 437 (2014) 547-561. doi 10 . 1093/mnras/stt 1907 arXiv:1307.7026

[103] R. K. Sheth, An excursion set model for the distribution of dark matter and dark matter haloes, MNRAS 300 (1998) 1057-1070. doi $10.1046 / j .1365-8711.1998 .01976 . x$ arXiv:astro-ph/9805319

[104] R. K. Sheth, H. J. Mo, G. Tormen, Ellipsoidal collapse and an improved model for the number and spatial distribution of dark matter haloes, MNRAS 323 (2001) 1-12. doi 10.1046/j.1365-8711. $2001.04006 . x$ arXiv:arXiv:astro-ph/9907024

[105] R. K. Sheth, G. Tormen, An excursion set model of hierarchical clustering: ellipsoidal collapse and the moving barrier, MNRAS 329 (2002) 61-75. doi $10.1046 / j .1365-8711.2002 .04950 . x$ arXiv:arXiv:astro-ph/0105113

[106] S. G. Murray, C. Power, A. S. G. Robotham, HMFcalc: An online tool for calculating dark matter halo mass functions, Astronomy and Computing 3 (2013) 23-34. doi $10.1016 / j . a s c o m .2013 .11 .001$ arXiv: 1306.6721

[107] W. H. Press, P. Schechter, Formation of Galaxies and Clusters of Galaxies by Self-Similar Gravitational Condensation, ApJ 187 (1974) 425438. doi $10.1086 / 152650$

[108] M. H. Abdullah, A. Klypin, G. Wilson, Cosmological Constraint on $\Omega_{m}$ and $\sigma_{8}$ from Cluster Abundances using the GalWCat19 OpticalSpectroscopic SDSS Catalog (2020). arXiv:2002.11907

[109] M. H. Abdullah, G. Wilson, A. Klypin, L. Old, E. Praton, G. Ali, GalWeight Application: A Publicly Available Catalog of Dynamical Parameters of 1800 Galaxy Clusters from SDSS-DR13, (GalWCat19), Astrophys. J. Suppl. 246 (2020) 2. doi $10.3847 / 1538-4365 / a b 536 e$ arXiv:1907.05061

[110] J. Hjorth, J. Oukbir, E. van Kampen, The M- $\mathrm{T}_{X}$ relation for clusters of galaxies, New Astronomy Reviews 42 (1998) 145-148. doi 10.1016/ S1387-6473(98)00037-2

[111] J. Hjorth, J. Oukbir, E. van Kampen, The mass-temperature relation for clusters of galaxies, MNRAS 298 (1998) L1-L5. doi $10.1046 / j$. 1365-8711.1998.01780.x arXiv:astro-ph/9802293

[112] L. Campanelli, G. L. Fogli, T. Kahniashvili, A. Marrone, B. Ratra, Galaxy cluster number count data constraints on cosmological parameters, European Physical Journal C 72 (2012) 2218. doi $10.1140 /$ epjc/s10052-012-2218-4 arXiv:1110.2310 